\title{
Posicionamento sobre Indicações da Ecocardiografia em Cardiologia Fetal, Pediátrica e Cardiopatias Congênitas do Adulto - 2020
}

Position Statement on Indications for Echocardiography in Fetal and Pediatric Cardiology and Congenital Heart Disease of the Adult - 2020

Realização: Departamento de Imagem Cardiovascular (DIC) da Sociedade Brasileira de Cardiologia (SBC) e Sociedad de Imágenes Cardiovasculares de Sociedad Interamericana de Cardiología (Sisiac, Siac)

Conselho de Normatizações e Diretrizes (2020-2021): Brivaldo Markman Filho, Antonio Carlos Sobral Sousa, Aurora Felice Castro Issa, Bruno Ramos Nascimento, Harry Correa Filho, Marcelo Luiz Campos Vieira

Coordenador de Normatizações e Diretrizes (2020-2021): Brivaldo Markman Filho

Editora Coordenadora: Samira Saady Morhy

Coeditores: Silvio Henrique Barberato, Carlos Eduardo Rochitte, Marcelo Luiz Campos Vieira

Autores do Posicionamento: Samira Saady Morhy, ${ }^{10}$ Silvio Henrique Barberato, ${ }^{2,3}$ (깅 Alessandro Cavalcanti Lianza, ${ }^{1,4,5}$ Andressa Mussi Soares, ${ }^{6 \mathbb{0}}$ Gabriela Nunes Leal, ${ }^{1,4,5,7}{ }^{\circledR}$ Ivan Romero Rivera, ${ }^{\circledR}{ }^{\circledR}$ Marcia Ferreira Alves Barberato, ${ }^{2}$ Vitor Guerra, ${ }^{9}{ }^{\circledR}$ Zilma Verçosa de Sá Ribeiro, ${ }^{10,11}$ Ricardo Pignatelli, ${ }^{12}$ Carlos Eduardo Rochitte, ${ }^{13}{ }^{\circledR 0}$ Marcelo Luiz Campos Vieira ${ }^{1,13}$

Hospital Israelita Albert Einstein, ${ }^{1}$ São Paulo, SP - Brasil

Cardioeco - Centro de Diagnóstico Cardiovascular, ${ }^{2}$ Curitiba, $P R-$ Brasil

Quanta Diagnóstico e Terapia, ${ }^{3}$ Curitiba, $P R-$ Brasil

Instituto da Criança e do Adolescente do Hospital das Clínicas da Faculdade de Medicina da Universidade de São Paulo (HC-FMUSP), ${ }^{4}$ São Paulo, SP - Brasil

Hospital do Coração, ${ }^{5}$ São Paulo, SP - Brasil

Hospital Evangélico de Cachoeiro de Itapemirim e Clínica CORImagem, ${ }^{6}$ Cachoeiro de Itapemirim, ES - Brasil

Hospital e Maternidade São Luiz Itaim, ${ }^{7}$ São Paulo, SP - Brasil

Santa Casa de Misericórdia de Maceió, ${ }^{8}$ AL - Brasil

The Hospital for Sick Children, ${ }^{9}$ Toronto - Canadá

Hospital Português, ${ }^{10}$ Salvador, BA - Brasil

Hospital Aliança, ${ }^{11}$ Salvador, BA - Brasil

Texas Children's Hospital, Baylor College of Medicine, ${ }^{12}$ Houston, Texas - EUA

Instituto do Coração da Faculdade de Medicina da Universidade de São Paulo (InCor, FMUSP), ${ }^{13}$ São Paulo, SP - Brasil

Este posicionamento deverá ser citado como:

Morhy SS, Barberato SH, Lianza AC, Soares AM, Leal GN, Rivera IR, et al. Posicionamento sobre Indicações da Ecocardiografia em Cardiologia Fetal, Pediátrica e Cardiopatias Congênitas do Adulto - 2020. Arq Bras Cardiol. 2020; 115(5):987-1005

Nota: Estes posicionamentos se prestam a informar e não a substituir o julgamento clínico do médico que, em última análise, deve determinar o tratamento apropriado para seus pacientes.

Correspondência: Sociedade Brasileira de Cardiologia - Av. Marechal Câmara, 360/330 - Centro - Rio de Janeiro - CEP: $20020-907$.

E-mail: diretrizes@cardiol.br

DOI: https://doi.org/10.36660/abc.20201122 
Declaração de potencial conflito de interesses dos autores/colaboradores do Posicionamento sobre Indicações da Ecocardiografia em Cardiologia Fetal, Pediátrica e Cardiopatias Congênitas do Adulto - 2020

Se nos últimos 3 anos o autor/colaborador do Posicionamento:

\begin{tabular}{|c|c|c|c|c|c|c|c|}
\hline $\begin{array}{l}\text { Nomes Integrantes } \\
\text { do Posicionamento }\end{array}$ & $\begin{array}{l}\text { Participou } \\
\text { de estudos } \\
\text { clínicos elou } \\
\text { experimentais } \\
\text { subvencionados } \\
\text { pela indústria } \\
\text { farmacêutica } \\
\text { ou de } \\
\text { equipamentos } \\
\text { relacionados ao } \\
\text { posicionamento } \\
\text { em questão }\end{array}$ & $\begin{array}{l}\text { Foi palestrante } \\
\text { em eventos } \\
\text { ou atividades } \\
\text { patrocinadas } \\
\text { pela indústria } \\
\text { relacionados ao } \\
\text { posicionamento } \\
\text { em questão }\end{array}$ & $\begin{array}{c}\text { Foi (é) membro } \\
\text { do conselho } \\
\text { consultivo } \\
\text { ou diretivo } \\
\text { da indústria } \\
\text { farmacêutica } \\
\text { ou de } \\
\text { equipamentos }\end{array}$ & $\begin{array}{l}\text { Participou } \\
\text { de comitês } \\
\text { normativos } \\
\text { de estudos } \\
\text { científicos } \\
\text { patrocinados } \\
\text { pela indústria }\end{array}$ & $\begin{array}{l}\text { Recebeu auxílio } \\
\text { pessoal ou } \\
\text { institucional da } \\
\text { indústria }\end{array}$ & $\begin{array}{l}\text { Elaborou textos } \\
\text { científicos em } \\
\text { periódicos } \\
\text { patrocinados } \\
\text { pela indústria }\end{array}$ & $\begin{array}{l}\text { Tem ações da } \\
\text { indústria }\end{array}$ \\
\hline $\begin{array}{l}\text { Alessandro } \\
\text { Cavalcanti Lianza }\end{array}$ & Não & Não & Não & Não & Não & Não & Não \\
\hline $\begin{array}{l}\text { Andressa Mussi } \\
\text { Soares }\end{array}$ & Não & Não & Não & Não & Não & Não & Não \\
\hline $\begin{array}{l}\text { Carlos Eduardo } \\
\text { Rochitte }\end{array}$ & Não & Não & Não & Não & Não & Não & Não \\
\hline Gabriela Nunes Leal & Não & Não & Não & Não & Não & Não & Não \\
\hline Ivan Romero Rivera & Não & Não & Não & Não & Não & Não & Não \\
\hline $\begin{array}{l}\text { Marcelo Luiz } \\
\text { Campos Vieira }\end{array}$ & Não & Não & Não & Não & Não & Não & No \\
\hline $\begin{array}{l}\text { Marcia Ferreira } \\
\text { Alves Barberato }\end{array}$ & Não & Não & Não & Não & Não & Não & Não \\
\hline Ricardo Pignatelli & Não & Não & Não & Não & Não & Não & Não \\
\hline Samira Saady Morhy & Não & Não & Não & Não & Não & Não & Não \\
\hline $\begin{array}{l}\text { Silvio Henrique } \\
\text { Barberato }\end{array}$ & Não & Não & Não & Não & Não & Não & Não \\
\hline Vitor C. Guerra & Não & Não & Não & Não & Não & Não & Não \\
\hline $\begin{array}{l}\text { Zilma Verçosa de Sá } \\
\text { Ribeiro }\end{array}$ & Não & Não & Não & Não & Não & Não & Não \\
\hline
\end{tabular}




\section{Posicionamento}

\section{Sumário}

1. Introdução 989

2. Ecocardiografia Fetal

3. Ecocardiografia no Recém-nascido 990

4. Ecocardiografia em Lactentes, Crianças e Adolescentes ...... 990

5. Ecocardiografia Pediátrica nas Cardiopatias Adquiridas ....... 993

6. Ecocardiografia em Adultos com Cardiopatia Congênita ...... 993

7. Ecocardiografia Transesofágica em Cardiologia Pediátrica... 993

7.1. Ecocardiografia Transesofágica como Ferramenta Diagnóstica .......... 994

7.2. Ecocardiografia Transesofágica no Intraoperatório

.994

7.3. Ecocardiografia Transesofágica na Unidade de Terapia Intensiva (UTI) ... 994

7.4. Ecocardiografia Transesofágica no Laboratório de Hemodinâmica...... 994

8. Ecocardiografia sob Estresse em Cardiologia Pediátrica....... 996

9. Ecocardiografia Tridimensional..... ... 996

10. Estudo da Deformação Miocárdica em Pacientes Pediátricos .. 998

10.1. Strain Ventricular em Cardiopatias Adquiridas na Infância . 999

10.2. Strain Ventricular em Cardiopatias Congênitas 1000

10.3. Strain Atrial Direito e Esquerdo em Pediatria 1001

10.4. Perspectivas de Utilização do Strain Ventricular no Feto 1001

Referênciass 1001

\section{Introdução}

De acordo com as "Normas para Elaboração de Diretrizes, Posicionamentos e Normatizações" sancionadas pela Sociedade Brasileira de Cardiologia, este documento foi escrito para atualizar as indicações da ecocardiografia em cardiologia fetal, pediátrica e cardiopatias congênitas do adulto, e complementa o posicionamento sobre indicações da ecocardiografia em adultos, recentemente publicado. 1 Tal posicionamento não pretende ser uma ampla revisão da ecocardiografia em cardiopatias congênitas, mas sim um guia básico indispensável para amparar a tomada de decisão clínica racional do médico que solicita o exame. Embora leve em consideração os significativos avanços tecnológicos recentes da ecocardiografia, sua finalidade não é descrever com detalhes os métodos ecocardiográficos, mas resumir de forma clara e concisa as principais situações em que a ecocardiografia traz benefício para o diagnóstico e/ou a orientação terapêutica nesses grupos de pacientes. Optou-se, neste manuscrito, por destacar a classe da indicação (grau de recomendação), conforme a descrição a seguir:

- Classe I: condições para as quais há evidências conclusivas ou, na sua falta, consenso geral de que o exame é útil e seguro.

- Classe II: condições para as quais há evidências conflitantes e/ou divergência de opinião sobre utilidade e/ou segurança do exame.

- Classe Ila: evidências ou opiniões favoráveis ao exame. A maioria dos especialistas aprova.

- Classe IIb: utilidade e/ou segurança menos bem estabelecidas, havendo opiniões divergentes.
- Classe III: condições para as quais há evidências ou consenso de que o exame não é útil e, em alguns casos, pode ser prejudicial.

Em adição, foi descrito também o nível de evidência:

- A: diversos estudos clínicos randomizados concordantes ou metanálises robustas.

- B: dados de metanálises menos robustas ou estudo clínico randomizado único ou estudos observacionais.

- C: opinião de especialistas.

Assim, convencionou-se que, em todas as tabelas com recomendação do emprego da ecocardiografia nos diferentes cenários clínicos, constam as colunas com classe de indicação e nível de evidência

\section{Ecocardiografia Fetal}

A incidência da cardiopatia congênita é estimada entre 6-12/1.000 nascidos vivos; ${ }^{2,3}$ entretanto, estima-se que sua prevalência na vida fetal seja maior. Vários fatores estão associados ao aumento do risco de cardiopatia congênita em fetos, como os fatores familiares, condições maternas e fetais. A ecocardiografia fetal é a principal ferramenta para o diagnóstico detalhado das patologias cardíacas, desde o final do primeiro trimestre até o termo. O período para realização da ecocardiografia fetal é determinado por múltiplos fatores, tais como o motivo de sua indicação e a idade gestacional na qual a alteração cardíaca e/ou extracardíaca foi detectada. A ecocardiografia no rastreamento de gestação de risco deve ser feita a partir de 18 a 22 semanas de gestação. Lembrando que esse rastreamento inicial pode não identificar lesões evolutivas ${ }^{4}$ e arritmias., ${ }^{5,6}$ Portanto, achados anômalos na rotina obstétrica devem ser prontamente encaminhados para nova ecocardiografia fetal.

O ecocardiograma fetal pode ser realizado em idades gestacionais mais precoces, incluindo o final do primeiro e o início do segundo trimestre, geralmente em gestações de alto risco para cardiopatias congênitas, principalmente na presença de translucência nucal aumentada no ultrassom morfológico do primeiro trimestre. ${ }^{7,8}$ Ecocardiograma fetal transabdominal permite a visibilização adequada das estruturas cardíacas na maioria das gestações entre 13 a 14 semanas, permitindo detecção de anomalias. No entanto, o ecocardiograma transvaginal é necessário se a realização do exame for antes de 13 semanas, devido ao tamanho reduzido das estruturas cardíacas e da distância entre o feto e a parede abdominal da mãe. ${ }^{7,8}$ Quando o ecocardiograma fetal é realizado antes de 18 semanas, o exame deve ser repetido entre 18 a 22 semanas de gestação, devido à limitação de resolução de imagem, podendo não diagnosticar algumas lesões cardíacas, e também ao potencial de progressão de lesões não detectadas em idade gestacional precoce. ${ }^{7-9}$

O período e a frequência do ecocardiograma devem ser guiados por: gravidade da lesão, sinais de insuficiência cardíaca, mecanismos de progressão e avaliação para o manejo perinatal.

As recomendações para a ecocardiografia fetal estão listadas nas Tabelas 1 e 2 . 
Tabela 1 - Recomendações para ecocardiografia fetal com perfil de gestação de risco elevado ${ }^{5-9}$

\begin{tabular}{|c|c|c|}
\hline Recomendações & $\begin{array}{l}\text { Grau de } \\
\text { recomendação }\end{array}$ & $\begin{array}{l}\text { Nível de } \\
\text { evidência }\end{array}$ \\
\hline DM pré-gestacional & I & A \\
\hline DMG diagnosticada no primeiro trimestre & II & B \\
\hline Fenilcetonúria maternal & I & A \\
\hline Anticorpos maternos SSA/SSB & Ila & B \\
\hline \multicolumn{3}{|l|}{ Medicacões maternas: } \\
\hline Inibidores ECA & Ila & B \\
\hline Ácido retinoico & I & B \\
\hline AINH no terceiro trimestre & 1 & A \\
\hline $\begin{array}{l}\text { Infecção materna por rubéola } \\
\text { no primeiro trimestre }\end{array}$ & I & C \\
\hline $\begin{array}{l}\text { Infecção materna com suspeita } \\
\text { de miocardite/pericardite }\end{array}$ & I & C \\
\hline Reprodução assistida & Ila & A \\
\hline $\begin{array}{l}\text { Cardiopatia congênita em } \\
\text { parente de primeiro grau }\end{array}$ & 1 & B \\
\hline $\begin{array}{l}\text { Cardiopatia com herança mendeliana em } \\
\text { parente de primeiro ou segundo grau }\end{array}$ & I & C \\
\hline $\begin{array}{l}\text { Suspeita de cardiopatia } \\
\text { no ultrassom obstétrico }\end{array}$ & I & B \\
\hline Anomalia extracardiaca fetal & I & B \\
\hline Feto com alteração cromossômica & I & C \\
\hline $\begin{array}{l}\text { Feto com taquicardia ou bradicardia ou } \\
\text { batimentos irregulares frequentes }\end{array}$ & I & C \\
\hline $\mathrm{TN}>95 \%$ & I & A \\
\hline Gemelaridade monocoriônica & 1 & A \\
\hline Feto com hidropisia ou derrames & I & B \\
\hline
\end{tabular}

Tabela 2 - Recomendações para ecocardiografia fetal com perfil de gestação de risco baixo $0^{5-9}$

\begin{tabular}{|c|c|c|}
\hline Recomendações & $\begin{array}{l}\text { Grau de } \\
\text { recomendação }\end{array}$ & $\begin{array}{l}\text { Nível de } \\
\text { evidência }\end{array}$ \\
\hline $\begin{array}{l}\text { Medicações maternas: } \\
\text { Anticonvulsivante } \\
\text { Lítio } \\
\text { Vitamina A } \\
\text { Inibidores de serotonina } \\
\text { AINH no primeiro e segundo trimestre }\end{array}$ & $\mathrm{llb}$ & B \\
\hline Cardiopatias em parentes de segundo grau & llb & B \\
\hline Alterações do cordão umbilical e placenta & llb & C \\
\hline Alteração venosa intra-abdominal fetal & $\| l b$ & C \\
\hline
\end{tabular}

AINH: anti-inflamatórios não hormonais. Adaptada de Donafrio et al. ${ }^{7}$

\section{Ecocardiografia no Recém-nascido}

Os recém-nascidos deixam uma situação de circulação em paralelo com baixa resistência vascular sistêmica e alta resistência vascular pulmonar na vida fetal para uma circulação em série, em que o débito cardíaco de ambos os ventrículos deve ser igual, na presença de uma alta resistência vascular sistêmica. Essas mudanças circulatórias que ocorrem após o nascimento podem demorar de dias a semanas para se completarem, principalmente nos prematuros, por causa de uma inabilidade de as comunicações presentes na vida fetal se fecharem prontamente. Assim, a persistência do canal arterial (PCA), a persistência de altas pressões pulmonares e a incapacidade do miocárdio imaturo de bombear sangue contra uma resistência vascular sistêmica, repentinamente alta, podem levar a uma redução transitória do fluxo sanguíneo sistêmico e alterar a hemodinâmica desses pacientes. ${ }^{9}$ Além disso, a presença de anomalias cardíacas estruturais ou de alterações extracardíacas, como sepse ou hérnia diafragmática, é tolerada de forma diferente nessa faixa etária. ${ }^{10}$

A fisiologia transicional da circulação cardiovascular no período neonatal faz com que esses pacientes precisem ser avaliados como um grupo à parte.

As razões mais comuns para realização do ecocardiograma no período neonatal são para reconhecer ou excluir doenças cardíacas congênitas estruturais em pacientes com: sopro cardíaco, alteração do teste do coraçãozinho, ${ }^{11}$ pacientes em choque, hipoxêmicos, em insuficiência respiratória ou com múltiplas malformações. Seguidas pelas anomalias funcionais, como avaliação da persistência do canal arterial, da hemodinâmica pulmonar e da função cardíaca (ver Tabela 2).

A avaliação ecocardiográfica de pacientes internados em unidades de terapia intensiva neonatal se justifica, inclusive de forma evolutiva, como um fator de mudanças específicas no manejo clínico do neonato.

As recomendações para a ecocardiografia em recémnascidos estão listadas na Tabela 3.

\section{Ecocardiografia em Lactentes, Crianças e Adolescentes}

Por ser um método não invasivo e prover informações anatômicas, hemodinâmicas e fisiológicas dos corações pediátricos, o ecocardiograma é o principal método diagnóstico para avaliação inicial das cardiopatias congênitas ou adquiridas em lactentes, crianças e adolescentes.

Crianças com doenças cardíacas representam um grupo variado de pacientes, frequentemente caracterizado por malformação anatômica complexa, que necessitam de seguimento durante toda a vida. Assim, estudos seriados podem ser indicados para monitoramento de função valvar, crescimento de estruturas cardiovasculares, função ventricular e seguimento de intervenções medicamentosas ou cirúrgicas. ${ }^{9,16-18}$

Sinais e sintomas como cianose, déficit de crescimento, dor torácica induzida por exercício, síncope, desconforto respiratório, sopros, insuficiência cardíaca, alteração de pulsos e cardiomegalia podem sugerir doença cardíaca estrutural. 


\section{Posicionamento}

\begin{tabular}{|c|c|c|}
\hline Recomendações & $\begin{array}{c}\text { Grau de } \\
\text { recomendação }\end{array}$ & $\begin{array}{l}\text { Nível de } \\
\text { evidência }\end{array}$ \\
\hline Sopro cardíaco patológico ou outras anormalidades na ausculta cardíaca & I & C \\
\hline Cianose central, insuficiência cardíaca, choque cardiogênico, desconforto respiratório & 1 & A \\
\hline Assimetria de pulsos e/ou gradiente de pressão arterial entre membros superiores e inferiores & I & A \\
\hline Cardiomegalia ao exame radiológico de tórax ou encontros anormais sugestivos de doença cardíaca & I & A \\
\hline Síndromes associadas à doença cardiovascular & I & B \\
\hline Anomalias extracardíacas & I & B \\
\hline Anomalia da posição cardíaca ou do situs & I & B \\
\hline Ecocardiograma fetal e/ou obstétrico alterado ou duvidoso para malformação cardíaca & 1 & C \\
\hline Cirurgia cardíaca corretiva ou paliativa & 1 & B \\
\hline História de hidropisia fetal & 1 & B \\
\hline Suspeita clínica de canal arterial patente & I & A \\
\hline Avaliação do significado hemodinâmico do PCA, seguimento dos efeitos da terapêutica & I & A \\
\hline Avaliação evolutiva do neonato submetido à cirurgia para fechamento de canal arterial com instabilidade hemodinâmica & I & A \\
\hline Asfixia perinatal com alteração hemodinâmica e/ou alteração de biomarcadores & 1 & A \\
\hline Suspeita de hipertensão pulmonar & I & A \\
\hline Avaliação evolutiva da hipertensão pulmonar em tratamento medicamentoso & I & A \\
\hline Hipotensão & I & A \\
\hline Avaliação da posição das cânulas do suporte de vida extracorpóreo, manutenção e desmame da ECMO & I & A \\
\hline Doença sistêmica materna associada à conhecida anomalia neonatal & Ila & B \\
\hline Infecção materna durante a gestação ou parto com potencial sequela cardíaca fetal ou neonatal & Ila & B \\
\hline Diabetes materna sem ecocardiograma fetal ou com ecocardiograma fetal normal & $11 \mathrm{~b}$ & B \\
\hline Fenilcetonúria maternal & I & A \\
\hline Disfunção autoimune materna & Ila & B \\
\hline Exposição materna a teratógenos & Ila & B \\
\hline Dificuldade de crescimento na ausência de anormalidades clínicas definidas & Ila & C \\
\hline História de ritmo cardíaco ectópico fetal não sustentado, na ausência de arritmia após o parto & III & C \\
\hline Acrocianose com saturação normal pelo oxímetro de pulso nas extremidades superiores e inferiores & III & C \\
\hline Avaliação morfológica e funcional no pós-operatório de cirurgia cardíaca & I & B \\
\hline Avaliar derrame pericárdico e impacto hemodinâmico e guiar procedimentos intervencionistas & 1 & A \\
\hline Identificar posição do cateter venoso central e suas complicações (trombose e infecção) & I & A \\
\hline
\end{tabular}

ECMO: oxigenação por membrana extracorpórea; PCA: persistência do canal arterial. 
Tabela 4 - Recomendações para ecocardiografia em lactentes, crianças e adolescentes , $^{9,12,16-18}$

\section{Recomendações}

\begin{tabular}{cc}
$\begin{array}{c}\text { Grau de } \\
\text { recomendação }\end{array}$ & $\begin{array}{c}\text { Nível de } \\
\text { evidência }\end{array}$ \\
\hline I & C \\
\hline I & B \\
\hline I & B \\
\hline I & B \\
\hline I & B \\
\hline I & C \\
\hline I & B \\
\hline I & B \\
\hline I & A \\
\hline I & A \\
\hline Ilb & C \\
\hline I & B \\
\hline I & B \\
\hline Ila & C \\
\hline I & B \\
\hline I & B \\
\hline
\end{tabular}

Sopro patológico ou outra evidência de anormalidade cardíaca

Anomalia da posição cardíaca ou do situs

Cardiomegalia ao exame radiológico de tórax ou encontros anormais sugestivos de doença cardíaca

Alteração ao eletrocardiograma

Avaliação pré-operatória imediata de cirurgia cardíaca

Mudança no quadro clínico de paciente com cardiopatia conhecida

Avaliação morfológica e funcional no pós-operatório de cirurgia cardíaca

História familiar de doença cardíaca transmitida geneticamente

Doença neuromuscular com envolvimento miocárdico

Sinais e sintomas de endocardite infecciosa

Sinais e sintomas de insuficiência cardíaca

Palpitações sem outros sintomas, história familiar benigna e eletrocardiograma normal

Palpitações com história familiar de arritmia, morte súbita, cardiomiopatia.

Palpitações em paciente com cardiomiopatia conhecida

Palpitações com eletrocardiograma anormal ou canulopatia conhecida

Assimetria de pulsos periféricos

Síndrome associada com doença cardiovascular; genótipo positivo para cardiomiopatia; anomalia cromossômica associada à doença cardiovascular

Determinação do momento adequado para tratamento clínico ou cirúrgico em pacientes com cardiopatia conhecida

Seleção, implantação, patência e monitoramento de dispositivos endovasculares.

Identificação de shunts intracardíacos e intravasculares antes, durante e após cateterismo cardíaco intervencionista percutâneo

Febre prolongada, sem causa aparente, em paciente com cardiopatia congênita

Sopro funcional em paciente assintomático

Retardo do crescimento na ausência de anormalidade clínica definida

I B

Dor torácica atípica, identificada como origem musculoesquelética em paciente assintomático

Síncope com eletrocardiograma anormal, síncope ao exercício

Síncope com história familiar de cardiomiopatia ou morte súbita

Síncope neurocardiogênica (vasovagal)

Dor torácica ao esforço ou dor torácica em repouso com eletrocardiograma anormal

Dor torácica associada à febre ou uso de drogas ilícitas

A

I A

I A

Ilb C

III

Sopro inocente presumível com sinais e sintomas de doença cardíaca

Cianose central

Deformidade da parede torácica e escoliose pré-operatória

Início, manutenção e desmame do suporte de vida extracorpóreo

Ecocardiograma normal prévio com mudança no estado cardiovascular e/ou nova história familiar sugestiva de doença cardíaca hereditária

Biomarcadores cardíacos anormais

Hemoglobinopatias

Doenças do tecido conectivo (Marfan, Loeys, Dietz e outras)

Distrofia muscular

Doenças autoimunes

Hipertensão arterial

Acidente vascular encefálico

Doença metabólica, mitocondrial ou de depósito

História familiar de doença cardiovascular: morte súbita antes dos 50 anos de idade, doenças do tecido conectivo (Marfan ou síndrome de Loeys Dietz), hipertensão arterial idiopática

História familiar de doença cardiovascular: cardiomiopatia hipertrófica, cardiomiopatia dilatada não isquêmica, hipertensão arterial pulmonar hereditária

\begin{tabular}{cc} 
I & A \\
\hline I & A \\
\hline lla & C \\
\hline I & B \\
\hline Ila & B \\
\hline I & C \\
\hline I & A \\
\hline Ilb & C \\
\hline I & B
\end{tabular}

lla $\quad \mathrm{C}$

I B

I B

I B

I B

I B

I $A$

I B

I B

lla C

Ila B 
Além disso, o ecocardiograma pode ser indicado mesmo sem quadro clínico específico em pacientes com história familiar de doença cardíaca hereditária, síndromes genéticas associadas à cardiopatia estrutural, exames complementares alterados (ecocardiografia fetal, radiografia de tórax e eletrocardiograma).

Pacientes com arritmias podem ter cardiopatia estrutural, como transposição corrigida das grandes artérias e anomalia de Ebstein, tumores cardíacos e cardiomiopatias. As arritmias sustentadas e o uso de medicações antiarrítmicas podem levar à alteração na função miocárdica, sendo importante a realização do ecocardiograma no manejo clínico desses pacientes.

As recomendações para a ecocardiografia em lactentes, crianças e adolescentes estão listadas na Tabela 4.

\section{Ecocardiografia Pediátrica nas Cardiopatias Adquiridas}

As cardiopatias adquiridas ocorrem principalmente em doenças sistêmicas associadas a processos inflamatórios, doenças renais, uso de quimioterápicos cardiotóxicos, doença parenquimatosa pulmonar e após transplante cardíaco.

O acometimento miocárdico pode ocorrer em várias condições, tais como doenças inflamatórias sistêmicas (principalmente naquelas com curso mais agressivo como lúpus eritematoso sistêmico juvenil, artrite idiopática juvenil e febre reumática). ${ }^{19-22}$ Durante a realização de quimioterapia cardiotóxica (principalmente os antracíclicos) e radioterapia em região mediastinal, o ecocardiograma está indicado antes, durante e após o tratamento, com intuito de indicar medidas cardioprotetoras e até mesmo mudar a terapêutica em alguns casos. ${ }^{23}$

Nos pacientes nefropatas crônicos/hipertensos e/ou dialíticos, o ecocardiograma fornece informações preciosas ao clínico no que diz respeito à geometria ventricular, função sistólica/diastólica e volemia. Isso, muitas vezes, orienta a mudança do esquema de diálise, assim como introdução ou troca de anti-hipertensivos e de fármacos vasoativos. ${ }^{24}$

Nos pacientes pneumopatas, o ecocardiograma viabiliza não apenas a estimativa de pressões pulmonares, mas também a avaliação do desempenho ventricular direito, que guarda importante correlação com o prognóstico clínico. ${ }^{25-27}$

Em crianças e adolescentes com AIDS, o ecocardiograma é utilizado para investigação de acometimento cardíaco direto pelo vírus, que podem causar miocardiopatia dilatada, hipertensão pulmonar e até hipertrofia ventricular, além dos efeitos de doenças oportunistas e/ou efeitos colaterais das medicações utilizadas. ${ }^{28}$

Com o número crescente de insuficiência cardíaca final em crianças, faz-se necessário avaliação pré e pós-transplante cardíaco e/ou cardiopulmonar ${ }^{29}$ além de auxiliar na tomada de decisão para introducão/retirada de suporte cardiovascular. ${ }^{30}$

As recomendações para a ecocardiografia em recémnascidos, lactentes, crianças e adolescentes com cardiopatia adquirida estão listadas na Tabela 5.

\section{Ecocardiografia em Adultos com Cardiopatia Congênita}

A cardiologia pediátrica experimentou notáveis avanços nos últimos 30 anos, tanto no aspecto diagnóstico com o advento da ecocardiografia quanto no tratamento de correção das cardiopatias, inicialmente cirúrgico e posteriormente por técnica percutânea na sala de hemodinâmica. Dados recentes indicam que, em 2010, a população estimada de adultos com cardiopatia congênita nos EUA era de 1,4 milhão de pacientes ${ }^{30}$ Esta população apresenta problemas relacionados com defeitos residuais, novos defeitos adquiridos, como o refluxo pulmonar após correção definitiva de tetralogia de Fallot, ou obstruções após cirurgia de Jatene, arritmias, insuficiência cardíaca, doença adquirida do adulto, endocardite infecciosa ou indicação de transplante cardíaco. Muitos sobrevivem com cirurgias paliativas que podem ou não precisar de correção definitiva, como Senning, Mustard, Rastelli, Glenn ou Fontan, que trazem novas complicações implícitas no método cirúrgico adotado e, ainda, muitos pacientes apresentam-se pela primeira vez, sem diagnóstico prévio da cardiopatia. ${ }^{32-35}$

Não há dúvida de que a ecocardiografia transtorácica bidimensional tem papel importante no diagnóstico e no acompanhamento dessas malformações. ${ }^{36}$ Avanços recentes como a ecocardiografia 3D mostraram superioridade na determinação de volumes e até mesmo da função ventricular, principalmente em malformações complexas como aquelas que apresentam fisiologia univentricular ou na avaliação do ventrículo direito, devendo ser empregadas sempre que houver disponibilidade e pessoal treinado para o seu uso. ${ }^{37}$ Ainda, a orientação cirúrgica da imagem em 3D permite melhor compreensão quando apresentadas ao cirurgião, o que possibilita um melhor planejamento cirúrgico. Da mesma forma, novas técnicas para a avaliação da função diastólica e da função segmentar como Doppler tecidual, strain ou strain rate podem ser de grande utilidade, principalmente nas condições com fisiologia univentricular ou naquelas com deformação das câmaras cardíacas, principalmente do ventrículo direito ${ }^{38}$ (ver nos itens 9 e 10).

A principal limitação da ecocardiografia na avaliação de adultos com cardiopatia congênita é a inadequada janela transtorácica em pacientes com cirurgia cardíaca prévia ou deformidades da parede torácica, assim como a ecocardiografia não é adequada na avaliação do arco aórtico, das artérias coronárias, das artérias pulmonares e dos vasos colaterais. Nessas situações, a ecocardiografia transesofágica, angiotomografia e a ressonância magnética (RM) são extremamente úteis.

As recomendações para a ecocardiografia em adultos com cardiopatias congênitas estão listadas na Tabela 6.

\section{Ecocardiografia Transesofágica em Cardiologia Pediátrica}

O ecocardiograma transesofágico (ETE) constitui uma via de acesso alternativa, por meio do uso de transdutores especiais, que fornece maior definição das estruturas cardíacas, aumentando as possibilidades diagnósticas do método.

É particularmente importante na definição de estruturas anatômicas complexas e anormalidades funcionais, nem sempre passíveis de avaliação pela ecocardiografia transtorácica isolada. 
Tabela 5 - Recomendações para ecocardiografia em recém-nascidos, lactentes, crianças e adolescentes com cardiopatia adquirida9,16-31

\begin{tabular}{|c|c|c|}
\hline Recomendações & $\begin{array}{l}\text { Grau de } \\
\text { recomendação }\end{array}$ & $\begin{array}{l}\text { Nível de } \\
\text { evidência }\end{array}$ \\
\hline $\begin{array}{l}\text { Avaliação inicial e reavaliações de pacientes com diagnóstico suspeito ou confirmado de síndrome de Kawasaki, arterite de Takayasu, } \\
\text { miopericardites, AIDS e febre reumática }\end{array}$ & I & B \\
\hline Pós-transplante cardíaco ou cardiopulmonar & I & B \\
\hline Avaliação inicial e reavaliações de pacientes em uso de quimioterápicos cardiotóxicos e com radioterapia mediastinal & I & B \\
\hline Avaliação inicial e reavaliações em pacientes com doença miocárdica & I & C \\
\hline Doença renal grave e/ou hipertensão arterial sistêmica para avaliação de acometimento cardíaco & I & B \\
\hline Avaliação de doadores para transplante cardíaco & I & $\mathrm{C}$ \\
\hline Hipertensão arterial pulmonar & I & A \\
\hline Avaliação evolutiva da hipertensão arterial pulmonar em terapia medicamentosa ou cirúrgica & I & $\mathrm{B}$ \\
\hline Início ou suspensão de suporte cardiopulmonar extracorpóreo & I & C \\
\hline Evento tromboembólico & I & C \\
\hline Sepse, insuficiência cardíaca direita ou cianose em paciente com cateter venoso & I & B \\
\hline Embolização sistêmica ou pulmonar em paciente com fluxo direita-esquerda e cateter venoso & I & C \\
\hline Síndrome de veia cava superior em paciente com cateter venoso & 1 & C \\
\hline Doença hepática & Ila & C \\
\hline Obesidade com outros fatores de risco cardiovasculares ou apneia obstrutiva do sono & Ila & C \\
\hline Sepse & Ila & B \\
\hline Fibrose cística sem evidência de cor pulmonale & Ila & C \\
\hline Acompanhamento de pacientes após febre reumática sem evidência de envolvimento cardíaco & $\mathrm{Ilb}$ & C \\
\hline Avaliação cardíaca após pericardite sem evidências de pericardite recorrente ou pericardite crônica & $\mathrm{Ilb}$ & C \\
\hline Febre em paciente com cateter venoso sem evidências de embolização sistêmica ou pulmonar & Ilb & C \\
\hline Avaliação de rotina para participação em esportes competitivos em pacientes com exame cardiovascular normal & Ilb & C \\
\hline Acompanhamento tardio de síndrome de Kawasaki sem evidências de anormalidades coronarianas na fase aguda & III & C \\
\hline Avaliação de rotina em paciente assintomático com cateter venoso & III & C \\
\hline
\end{tabular}

Com os avanços tecnológicos e miniaturização das sondas, a adoção do ETE vem se ampliando no campo da cardiologia pediátrica, podendo oferecer importantes informações para os pacientes desde a faixa etária neonatal até adolescentes e adultos, tanto no diagnóstico, na avaliação intraoperatória, no pós-operatório imediato e tardio e na unidade de terapia intensiva quanto no laboratório de hemodinâmica, auxiliando a realização de procedimentos intervencionistas.

\subsection{Ecocardiografia Transesofágica como Ferramenta Diagnóstica}

O ETE deve ser adotado para melhor definição diagnóstica da cardiopatia, nas situações em que é necessária melhor avaliação anatômica em algumas cardiopatias congênitas específicas, na maioria das vezes em adultos, visto que a qualidade da imagem em crianças é geralmente boa ao exame transtorácico (Tabela 7).

\subsection{Ecocardiografia Transesofágica no Intraoperatório}

O grande impacto da ecocardiografia transesofágica na sala cirúrgica é a detecção de defeitos residuais importantes que muitas vezes não são suspeitados. Vários autores têm demonstrado retorno à circulação extracorpórea para revisão cirúrgica após a realização do ETE intraoperatório com taxas variando de 6 a 11,4\% dos casos, nas diferentes séries avaliadas. ${ }^{46}$

As indicações de ETE intraoperatório para cardiopatias congênitas estão listadas na Tabela 8.

\subsection{Ecocardiografia Transesofágica na Unidade de Terapia Intensiva (UTI)}

A definição das imagens pelo ETT no pós-operatório imediato pode estar prejudicada pela presença de drenos, curativos, telas e ventilação mecânica, sendo necessária a adoção do ETE, que pode fornecer informações anatômicas (lesões residuais) e hemodinâmicas importantes na condução clínica e na terapêutica dos pacientes (Tabela 9).

\subsection{Ecocardiografia Transesofágica no Laboratório de Hemodinâmica}

O ETE auxilia a intervenção hemodinâmica detalhando o diagnóstico em diversas cardiopatias, monitorando o procedimento, além de fornecer informações anatômicas do resultado e de eventuais lesões residuais ${ }^{47}$ (Tabela 10). 


\section{Posicionamento}

\begin{tabular}{|c|c|c|}
\hline Recomendações & $\begin{array}{l}\text { Grau de } \\
\text { recomendação }\end{array}$ & $\begin{array}{l}\text { Nível de } \\
\text { evidência }\end{array}$ \\
\hline $\begin{array}{l}\text { Avaliação estrutural e funcional inicial na suspeita de cardiopatia congênita evidenciada por sopro, cianose, insaturação arterial, } \\
\text { anormalidade ao eletrocardiograma ou radiografia de tórax }\end{array}$ & 1 & C \\
\hline Mudança no quadro clínico em paciente com cardiopatia congênita conhecida, operada ou não & 1 & C \\
\hline $\begin{array}{l}\text { Dúvidas do diagnóstico original ou anormalidades estruturais ou hemodinâmicas não esclarecidas em paciente com cardiopatia } \\
\text { congênita conhecida }\end{array}$ & 1 & C \\
\hline Acompanhamento de pacientes com comunicação interventricular para avaliação de modificações morfológicas evolutivas & 1 & C \\
\hline $\begin{array}{l}\text { Acompanhamento periódico de pacientes com cardiopatia congênita, operada ou não, nos quais é necessária a avaliação da função } \\
\text { contrátil, valvar e de condutos }\end{array}$ & 1 & C \\
\hline $\begin{array}{l}\text { Acompanhamento anual em pacientes no pós-operatório de correção total, parcial ou paliativa, com defeitos residuais e sequelas que } \\
\text { possam comprometer a evolução clínica do paciente }\end{array}$ & 1 & C \\
\hline Identificação da origem e curso inicial das artérias coronárias & I & C \\
\hline Avaliação da síncope pós-exercício não explicada para definição diagnóstica inicial & I & C \\
\hline $\begin{array}{l}\text { Avaliação de lesão aórtica em pacientes com suspeita ou confirmação de síndrome de Marfan para avaliação seriada da aorta e/ou da } \\
\text { valva mitral }\end{array}$ & I & C \\
\hline $\begin{array}{l}\text { Exames periódicos em pacientes operados de PCA, CIA, CIV, coarctação aórtica ou valva aórtica bicúspide, sem defeito residual e sem } \\
\text { mudanças na condição clínica }\end{array}$ & III & C \\
\hline Acompanhamento de pacientes com cardiopatias sem significado hemodinâmico e sem mudança na condição clínica & III & C \\
\hline $\begin{array}{l}\text { Avaliação de lesões do arco aórtico, artérias pulmonares e colaterais, cuja anatomia é melhor definida por outros métodos } \\
\text { de diagnóstico }\end{array}$ & III & C \\
\hline $\begin{array}{l}\text { Avaliação periódica de malformação cardíaca sem alteração no exame físico, na condição clínica do paciente ou em outros exames } \\
\text { como eletrocardiograma e radiografia de tórax }\end{array}$ & III & C \\
\hline CIA: comunicação interatrial; CIV: comunicação interventricular; PCA: persistência do canal art & III & C \\
\hline
\end{tabular}

Tabela 7 - Recomendações para a ecocardiografia transesofágica como ferramenta diagnóstica9,45

\begin{tabular}{|c|c|c|}
\hline Recomendações & $\begin{array}{l}\text { Grau de } \\
\text { recomendação }\end{array}$ & $\begin{array}{l}\text { Nível de } \\
\text { evidência }\end{array}$ \\
\hline Confirmação ou exclusão de uma suspeita diagnóstica clínica relevante não demonstrada pelo ETT & 1 & A \\
\hline $\begin{array}{l}\text { Informações anatômicas e hemodinâmicas insuficientes pelo ETE, principalmente em crianças com deformidades torácicas ou com } \\
\text { obesidade e em adultos com cardiopatias congênitas }\end{array}$ & I & A \\
\hline $\begin{array}{l}\text { Avaliação de forame oval patente (FOP) como possivel etiologia de eventos embólicos centrais ou periféricos em pacientes jovens (< } \\
60 \text { anos), com contraste salino agitado para determinar a possibilidade de fluxo direita-esquerda. Avaliar fatores de risco do FOP para } \\
\text { AVE/AIT*: aneurisma de septo interatrial, passagem de um número > } 30 \text { microbolhas do átrio direito para o átrio esquerdo, túnel do } \\
\text { FOP > } 10 \text { mm e valva de Eustáquio proeminente }\end{array}$ & I & A \\
\hline Na avaliação de forame oval patente pré-implante de marca-passo transvenoso & I & A \\
\hline $\begin{array}{l}\text { Classificação, dimensão e localização do defeito septal atrial, principalmente em pacientes adultos e naqueles com definição } \\
\text { transtorácica inadequada para seleção de possíveis candidatos à oclusão percutânea e escolha da prótese. }\end{array}$ & I & A \\
\hline Avaliação de dissecção da aorta nas síndromes de Marfan, Ehlers-Danlos, Turner e na coartação de aorta & I & A \\
\hline Avaliação da aorta na arterite de Takayasu & I & A \\
\hline Avaliação dos tubos intra ou extracardíacos no pós-operatório de cirurgia de Senning, Mustard ou Fontan & I & A \\
\hline Na avaliação de trombos, massas, vegetações, abscessos e próteses & I & A \\
\hline Na determinação do grau e mecanismos de refluxo valvar mitral para auxiliar reparo cirúrgico ou percutâneo (Mitraclip) & 1 & $\mathrm{~B}$ \\
\hline
\end{tabular}

AVE/AIT: acidente vascular encefálico/ataque isquêmico transitório; ETE: ecocardiograma transesofágico; ETT: ecocardiograma transtorácico. 
Tabela 8 - Recomendações para a ecocardiografia transesofágica no intraoperatório 9,45-46

\begin{tabular}{|c|c|c|}
\hline Recomendação & $\begin{array}{l}\text { Grau de } \\
\text { recomendação }\end{array}$ & $\begin{array}{l}\text { Nível de } \\
\text { evidência }\end{array}$ \\
\hline Avaliação perioperatória da anatomia e da função cardíaca & I & A \\
\hline $\begin{array}{l}\text { Monitoramento de procedimentos cirúrgicos com riscos de fluxos anormais, refluxos valvares, obstruções residuais ou disfunção } \\
\text { miocárdica ventricular }\end{array}$ & I & A \\
\hline Cirurgias minimamente invasivas, guiadas por vídeo e procedimentos híbridos & 1 & A \\
\hline
\end{tabular}

Tabela 9 - Recomendações para a ecocardiografia transesofágica na UT $\left.\right|^{9,45}$

\begin{tabular}{ll}
\hline Recomendação & $\begin{array}{c}\text { Nível de } \\
\text { evidência }\end{array}$ \\
\hline Avaliação de defeitos residuais, derrame pericárdico, função ventricular em pacientes com má qualidade de janela transtorácica & $\begin{array}{c}\text { Grau de } \\
\text { recomendação }\end{array}$ \\
\hline Monitoramento pós-operatório em paciente com esterno aberto & A \\
\hline
\end{tabular}

Tabela 10 - Recomendações para a ecocardiografia transesofágica no laboratório de hemodinâmica ${ }^{9,45,47}$

\begin{tabular}{|c|c|c|}
\hline Recomendação & $\begin{array}{c}\text { Grau de } \\
\text { recomendação }\end{array}$ & $\begin{array}{c}\text { Nível de } \\
\text { evidência }\end{array}$ \\
\hline No fechamento percutâneo de forame oval patente, comunicações interatriais e interventriculares & 1 & A \\
\hline No fechamento de fenestrações em pós-operatório de cirurgia de Fontan & I & A \\
\hline Nas dilatações das tunelizações das cirurgias de Senning e de Mustard & I & A \\
\hline Nas estenoses de artérias pulmonares e de tubos com colocação de stents & $\mathrm{Ilb}$ & B \\
\hline Orientação na valvoplastia mitral e reparo valvar mitral percutâneo (Mitraclip) & I & A \\
\hline Orientação nas valvoplastias pulmonar e aórtica & Ila & A \\
\hline Implante de endopróteses aórticas para tratamento de aneurismas, dissecções, hematomas ou úlceras parietais da aorta torácica & I & A \\
\hline Orientação do cateter na perfuração e dilatação percutânea de valvas atrésicas & I & A \\
\hline Durante cateterismo intervencionista terapêutico para ablação por radiofrequência & I & A \\
\hline
\end{tabular}

\section{Ecocardiografia sob Estresse em Cardiologia Pediátrica}

A ecocardiografia sob estresse (físico ou farmacológico) é uma técnica bem estabelecida em adultos. ${ }^{48,49} \mathrm{Na}$ faixa etária pediátrica, não temos ainda uma diretriz ou recomendação específica; no entanto, como na população adulta, a aplicação em crianças e adolescentes tem sido mais concentrada na pesquisa de doença isquêmica, ${ }^{50-56}$ mas está em expansão para outras áreas de aplicação não necessariamente isquêmica ${ }^{55-63}$ (Tabela 7).

Ambos os tipos de estresse, farmacológico e exercício, podem ser aplicados em crianças, com algumas peculiaridades. ${ }^{64-66} \mathrm{~A}$ dobutamina é o agente farmacológico mais comum e usado nos mesmos protocolos dos pacientes adultos. Em geral, em crianças abaixo de 8 anos de idade, recomenda-se sedação ou até mesmo anestesia. O exercício físico pode ser usado em crianças acima de 8 anos de idade que são cooperativas e com habilidades para se exercitar, em esteira ou bicicleta. ${ }^{67}$

\section{Ecocardiografia Tridimensional}

A ecocardiografia tridimensional (3D) tem sido incorporada na prática clínica, adicionando informações ao ecocardiograma bidimensional (2D), principalmente para a abordagem dos defeitos congênitos nos quais a visão tridimensional oferece uma visão muito próxima dos planos anatômicos e cirúrgicos. ${ }^{68}$ Esse mesmo conceito se aplica aos procedimentos feitos no laboratório de hemodinâmica, nos quais a visão tridimensional pode não só guiar os procedimentos, mas também avaliar de melhor forma a anatomia para adequar aos dispositivos usados. Avaliação dos volumes ventriculares e função também tem sido alvo da tecnologia 3D, principalmente por avaliar a geometria ventricular nas mais diversas formas nos defeitos congênitos, incluindo os corações univentriculares. ${ }^{69,70} \mathrm{~A}$ avaliação das valvas atrioventriculares é possível não só do ponto de vista do detalhamento anatômico, incluindo o aparelho subvalvar, como também a avaliação funcional da movimentação do anel valvar, interação entre o movimento dos folhetos valvares e cordas. ${ }^{71}$ 
Quando se trata de paciente pediátrico, temos uma grande vantagem pela melhor janela acústica transtorácica, e, mais recentemente, houve um avanço no desenvolvimento de transdutores com um foot print menor e com frequência mais alta (2 a $8 \mathrm{Mhz}$ ). Entretanto, ainda não temos a mesma qualidade de imagem quando se usa a combinação 2D-3D com o mesmo transdutor, principalmente em pacientes pequenos. Outro grande desafio ainda a ser ultrapassado é o desenvolvimento do transdutor transesofágico pediátrico, o que limita o uso do ETE 3D em pacientes acima de $30 \mathrm{~kg}$ de acordo com as recomendações dos fabricantes. Em crianças pequenas, recomenda-se o uso de transdutores pediátricos com frequência mais alta, e também o ecocardiograma epicárdico, nos casos realizados no intraoperatório. O ecocardiograma 3D transesofágico deve ser sempre considerado em pacientes maiores (geralmente acima de 30 kg) se o 3D transtorácico não oferecer informação suficiente para o planejamento cirúrgico ou intervenção.

Existe uma variedade de defeitos congênitos nos quais o ecocardiograma 3D pode adicionar informações sobre as mais diversas estruturas anatômicas, incluindo septos atrial e ventricular, as valvas semilunares e atrioventriculares, assim como as vias de saída. Como tem havido um progresso tecnológico, a aplicação está se estendendo à medida que ocorre esse progresso e adequação para população pediátrica. Atualmente, o uso baseia-se mais na necessidade clínica de informações adicionais do que em estudos randomizados sobre a vantagem do 3D sobre o 2D. Portanto, é individualizado e de acordo com o perfil do laboratório de imagem ou hospital adotar a tecnologia para lesões específicas.

As lesões valvares e os defeitos septais isolados são as principais indicações; contudo, em situações em que há concomitante anomalia de conexão ventriculoarterial, como nas duplas vias de saída do ventrículo direito, a posição e o tamanho da comunicação interventricular podem ser melhor visualizados e demonstrados pelo ecocardiograma 3D.

De acordo com a área ou estrutura avaliada pelo ecocardiograma 3D transtorácico e/ou transesofágico, podemos ter informações relevantes que complementariam aquelas obtidas pelo ecocardiograma 2D. ${ }^{72-83}$ As artérias pulmonares, a valva pulmonar e até mesmo a via de saída do ventrículo direito, assim como o arco aórtico, adicionam pouca informação quando avaliadas pelo 3D (Tabela 12).

O ecocardiograma 3D também adiciona informação no contexto de algumas cardiopatias congênitas específicas em que existem anomalias de conexão (atrioventricular ou ventriculoarterial) ${ }^{76,84-86}$ (Tabela 13).

A aplicação do ecocardiograma 3D na sala de hemodinâmica para fechamento de defeitos dos septos atrial e ventricular complementa as imagens 2D para delimitar as bordas dos defeitos e estruturas relacionadas, ${ }^{87,88}$ especificamente nas comunicações atriais tipo Ostium Secundum, que são muito bem demonstradas por imagem em tempo real pelo ecocardiograma 3D transesofágico. O fechamento das comunicações interventriculares por dispositivos percutâneos ou transmural também pode ser guiado pelo ecocardiograma 3D, principalmente para avaliar estruturas próximas, como, por exemplo, folhetos e/ou cordas da valva tricúspide. Existem outras aplicações na sala de hemodinâmica no qual o ecocardiograma 3D pode guiar os procedimentos: fechamento das fenestrações na cirurgia de Fontan, fistulas coronárias, rupturas do seio de Valsalva, regurgitação paravalvar, perfuração de septo e localização dos eletrodos no processo de ressincronização ventricular. ${ }^{89-94}$

Um dos grandes desafios em cardiopatias congênitas é a avaliação dos volumes e função ventricular, por razões intrínsecas dos defeitos congênitos (posição cardíaca, anomalias de conexão, material não contrátil e diferenças de pré-carga ventricular, entre outras). Os softwares disponíveis foram desenvolvidos a partir da geometria ventricular esquerda de corações normais, o que muitas vezes invalida as informações obtidas pelo 3D. Apesar de as medidas de volumes e fração de ejeção serem replicáveis, o ecocardiograma 3D tem mostrado valores menores que a RM na quantificação dos volumes, o que impede de substituir um pelo outro. Portanto, a aplicação clínica ainda tem sido dificultada por ausência de valores de normalidade na população pediátrica. Não se recomenda o uso dos softwares desenvolvidos para ventrículo esquerdo ou direito

Tabela 11 - Recomendações para ecocardiografia sob estresse em cardiologia pediátrica

\begin{tabular}{|c|c|c|}
\hline Recomendação & $\begin{array}{l}\text { Grau de } \\
\text { recomendação }\end{array}$ & $\begin{array}{l}\text { Nível de } \\
\text { evidência }\end{array}$ \\
\hline Pesquisa de insuficiência coronária em crianças pós-transplante cardíaco tardio & Ila & B \\
\hline Avaliação tardia na doença de Kawasaki com alterações coronarianas na fase aguda & Ila & B \\
\hline Pós-operatório de cirurgia de Jatene, pós-operatório de origem e trajetos anormais das artérias coronárias e fístulas coronário-cavitárias & Ila & B \\
\hline Função ventricular nas miocardiopatias e nas insuficiências valvares mitral e aórtica & Ila & B \\
\hline $\begin{array}{l}\text { Rastreamento de disfunção ventricular em pacientes que recebem quimioterapia com antraciclinas e no pós-transplante, a fim de } \\
\text { avaliar função miocárdica durante o exercício }\end{array}$ & Ila & B \\
\hline $\begin{array}{l}\text { Pesquisa de insuficiência coronária em crianças com atresia pulmonar com septo ventricular íntegro, dislipidemia, diabetes melito } \\
\text { insulinodependente, estenose aórtica supravalvar }\end{array}$ & $\mathrm{Ilb}$ & B \\
\hline Avaliação do comportamento do gradiente de pressão em cardiomiopatia hipertrófica, estenoses valvares pulmonar e aórtica & Ilb & $\mathrm{B}$ \\
\hline $\begin{array}{l}\text { Avaliação da reserva miocárdica em pós-operatório tardio de cirurgias em plano atrial para transposição dos grandes vasos, avaliação } \\
\text { do ventrículo direito em pós-operatório tardio de tetralogia de Fallot }\end{array}$ & $\mathrm{Ilb}$ & $\mathrm{B}$ \\
\hline
\end{tabular}




\begin{tabular}{|c|c|c|c|c|}
\hline $\begin{array}{l}\text { Estrutura } \\
\text { anatômica de } \\
\text { interesse }\end{array}$ & Modalidade & Informações adicionais & Grau de recomendação & $\begin{array}{l}\text { Nivel de } \\
\text { evidência }\end{array}$ \\
\hline Septo interatrial & ETT/ETE & Dimensão, formato, localização do(s) defeito(s) & $\begin{array}{l}\text { I- Defeitos complexos ou residuais } \\
\text { II - Defeito central e único }\end{array}$ & $\begin{array}{l}B \\
B\end{array}$ \\
\hline Valva tricúspide & ETT/ETE & $\begin{array}{l}\text { Morfologia dos folhetos, aparelho subvalvar (cordas), } \\
\text { localização dos jatos regurgitantes }\end{array}$ & 1 & B \\
\hline Valva mitral & ETT/ETE & $\begin{array}{l}\text { Morfologia dos folhetos, aparelho subvalvar (cordas), } \\
\text { localização dos jatos regurgitantes }\end{array}$ & I & B \\
\hline Septo interventricular & ETT/ETE & Dimensão, formato, localização do(s) defeito(s) complexo (s) & I & B \\
\hline Via de saída do VE & ETT/ETE & Morfologia da obstrução subaórtica & 1 & B \\
\hline Valva aórtica & ETT/ETE & $\begin{array}{c}\text { Medida da valva aórtica, morfologia dos folhetos, } \\
\text { mecanismo de regurgitação }\end{array}$ & 1 & B \\
\hline Via de saída do VD & ETT/ETE & Morfologia e visualização do local de obstrução & III & C \\
\hline Valva pulmonar & ETT & Morfologia & Ila & C \\
\hline
\end{tabular}

ETT: ecocardiograma transtorácico; ETE: ecocardiograma transesofágico; VD: ventrículo direito; VE: ventrículo esquerdo.

Tabela 13 - Informações adicionais do ecocardiograma 3D em defeitos congênitos e recomendações ${ }^{71,79,83-86}$

\begin{tabular}{|c|c|c|c|c|}
\hline $\begin{array}{l}\text { Cardiopatia } \\
\text { congênita }\end{array}$ & Modalidades & Informações adicionais & $\begin{array}{c}\text { Grau de } \\
\text { recomendação }\end{array}$ & $\begin{array}{l}\text { Nível de } \\
\text { evidência }\end{array}$ \\
\hline DSAV & ETT/ETE & $\begin{array}{c}\text { Dimensão do defeito atrial e/ou ventricular; morfologia dos folhetos e do aparelho } \\
\text { subvalvar; avaliação do(s) jato(s) regurgitante(s); dimensões dos orifícios e ventrículos } \\
\text { nos defeitos desbalanceados }\end{array}$ & I & B \\
\hline $\begin{array}{l}\text { Conexão AV } \\
\text { discordante }\end{array}$ & ETT/ETE & $\begin{array}{l}\text { Morfologia e função das valvas tricúspide e mitral, localização e dimensão da CIV associada; } \\
\text { morfologia das vias de saída do VD e VE }\end{array}$ & 1 & B \\
\hline TGA complexas & ETT/ETE & $\begin{array}{l}\text { Morfologia e função das valvas tricúspide e mitral, localização e dimensão da CIV, anatomia das } \\
\text { vias de saída do VD e VE nos casos de obstrução }\end{array}$ & I & B \\
\hline $\begin{array}{l}\text { Tetralogia de } \\
\text { Fallot }\end{array}$ & ETT & Dimensão e localização da CIV e anatomia da via de saída do VD & III & C \\
\hline $\begin{array}{l}\text { Truncus } \\
\text { Arteriosus }\end{array}$ & ETT/ETE & Morfologia da valva truncal ${ }^{*}$ & III & C \\
\hline $\begin{array}{l}\text { Dupla via de } \\
\text { saída do VD }\end{array}$ & ETT & Relação das valvas atrioventriculares, da posição e do tamanho da CIV com as grandes artérias & III & C \\
\hline
\end{tabular}

AV: atrioventricular; CIV: comunicação interventricular; DSAV: defeito do septo atrioventricular; ETT: ecocardiograma transtorácico; ETE: ecocardiograma transesofágico; TGA: transposição das grandes artérias. "Especificamente para avaliação da valva truncal em pacientes mais velhos.

normais em ventrículos congenitamente malformados até que novos softwares ou modelos tenham sido validados. ${ }^{70,95-97}$

A recomendação geral para o uso do ecocardiograma 3D transtorácico em pediatria é que deve ser a de acordo com o tipo de paciente e com o perfil do laboratório de ecocardiograma e/ou hospital.

Existe o consenso de que o 3D é uma modalidade que complementa o ecocardiograma 2D, e não uma substituição, independentemente do tipo de lesão.

\section{Estudo da Deformação Miocárdica em Pacientes Pediátricos}

A deformação miocárdica (strain) vem se mostrando ferramenta útil na avaliação da função diastólica e sistólica, tanto em adultos como na população pediátrica. ${ }^{98} \mathrm{O}$ estudo do strain miocárdico pelo speckle-tracking é um método independente do ângulo de insonação e apresenta baixa variação intra e interobservador, o que permite quantificar a função ventricular global e regional de forma mais acurada do que os métodos tradicionais, como Doppler tecidual, fração de encurtamento ou de ejeção. ${ }^{99}$ Alguns estudos já demonstraram elevado valor prognóstico do strain obtido pelo speckle-tracking, reforçando sua utilidade tanto em patologias congênitas como adquiridas. ${ }^{100}$

No entanto, o strain miocárdico está sujeito a variações fisiológicas causadas por idade, sexo, frequência cardíaca, pré-carga, pressão arterial e superfície corpórea, além do tipo de software utilizado para análise. ${ }^{101} \mathrm{Um}$ esforço contínuo vem sendo feito no sentido de estabelecer valores normais de strain que possam ser utilizados como referência universal em pediatria, para que a avaliação da deformação miocárdica seja 
incorporada aos guidelines e comece a ser adotada na rotina clínica. ${ }^{102-104}$ Por ora, o estudo da deformação miocárdica nas diversas doenças pediátricas tem grau de recomendação II e nível de evidência B.

\subsection{Strain Ventricular em Cardiopatias Adquiridas na Infância}

A avaliação do strain ventricular direito e esquerdo é particularmente útil em situações nas quais se pretende identificar disfunção sistólica e/ou diastólica em fase subclínica. As informações obtidas através da análise do strain possibilitam intervenção terapêutica oportuna em diversas doenças sistêmicas que cursam com acometimento miocárdico.

A detecção precoce de lesão miocárdica secundária ao uso de antracíclicos é uma das mais relevantes contribuições do estudo da deformação miocárdica até o momento, já tendo sido incorporada em protocolos de acompanhamento de pacientes oncológicos. ${ }^{105-108}$

Já foi demonstrada a correlação entre o grau de atividade inflamatória e os valores de strain e de strain rate sistólico e diastólico do VE em pacientes com doenças reumatológicas, como lúpus eritematoso sistêmico juvenil. ${ }^{20}$

Outros trabalhos comprovaram a eficácia do strain obtido pela técnica de speckle-tracking na detecção de miocardite, não só de etiologia autoimune como também viral. ${ }^{109,110} \mathrm{O}$ padrão de comprometimento regional do strain de VE, nos casos de miocardiopatia dilatada em crianças, influencia a evolução para óbito ou para transplante, como demonstrado por Forsha et al., ${ }^{111}$ Outra utilidade do strain nos casos de miocardiopatia dilatada é detectar dissincronia, identificando os casos que podem se beneficiar de terapia de ressincronização. ${ }^{111}$

O estudo com strain pós-transplante cardíaco ortotópico em crianças pode identificar, com razoável sensibilidade e especificidade, os indivíduos que irão manifestar doença vascular do enxerto nos anos subsequentes. ${ }^{112}$ Alguns relatos, incluindo pequeno número de crianças transplantadas, sugere associação entre a redução de strain segmentar e a presença de rejeição em biópsias endomiocárdicas, o que favorece a técnica como instrumento diagnóstico menos invasivo em um futuro próximo. ${ }^{113-115}$

Em pacientes jovens com distrofia muscular de Duchenne, estudos demonstraram redução significativa do strain longitudinal e radial das paredes inferolateral e anterolateral do VE, mesmo antes do comprometimento da fração de ejeção ou do surgimento de sintomas de insuficiência cardíaca. ${ }^{116}$ Vários trabalhos têm demonstrado melhora do desempenho cardiovascular e da sobrevida em 10 anos dos pacientes com Duchenne que passam a receber inibidores da enzima de conversão da angiotensina e betabloqueadores já aos primeiros sinais ecocardiográficos de deterioração miocárdica, quando ainda são assintomáticos do ponto de vista cardiovascular. ${ }^{117}$

O estudo do strain miocárdico também tem contribuído para detecção de comprometimento miocárdico em doenças de depósito, como as mucopolissacaridoses (MPS) ${ }^{118}$ e a doença de Pompe. ${ }^{119}$ Trabalhos têm centrado atenção no strain miocárdico como parâmetro de avaliação do impacto da reposição enzimática a longo prazo sobre a função ventricular dos portadores dessas doenças. ${ }^{120}$

A análise do strain miocárdico também surge como uma possibilidade de diagnóstico precoce de inflamação miocárdica e disfunção ventricular nos casos de doença de Kawasaki. ${ }^{51}$ McCandless et al. ${ }^{121}$ evidenciaram redução do strain longitudinal de VE ao ecocardiograma inicial de pacientes com Kawasaki, que posteriormente vieram a desenvolver dilatação coronariana ou que mostraram resistência ao tratamento com imunoglobulina. Esses achados sugerem que o strain de VE pode vir a ser utilizado em breve como ferramenta de estratificação de risco em Kawasaki. ${ }^{121}$

Nos casos de disfunção miocárdica induzida por sepse pediátrica, o strain longitudinal e circunferencial do VE parecem já estar reduzidos em fases iniciais, a despeito da fração de ejeção ainda conservada. ${ }^{122}$

Em pacientes adultos com insuficiência renal crônica (IRC), já foi comprovada a redução do strain longitudinal do VE, mesmo em estágios iniciais da doença e ainda com fração de ejeção preservada. Atribui-se o comprometimento precoce da deformação miocárdica à fibrose induzida por inflamação crônica e por toxinas urêmicas. Além disso, a disfunção endotelial que acompanha a IRC pode acarretar resposta vasodilatadora inadequada, causando isquemia em um ventrículo já hipertrófico. Achados semelhantes já foram documentados também em populações pediátricas, restando estabelecer se essa redução do strain longitudinal do VE pode ser utilizada como um preditor específico de morbidade e mortalidade em crianças com IRC. ${ }^{123}$

Alterações cardiovasculares são comuns em indivíduos infectados pelo HIV, mas frequentemente são subdiagnosticadas e não tratadas, o que impacta na qualidade de vida e na mortalidade a longo prazo. São atribuídas tanto ao efeito direto do vírus quanto às medicações antirretrovirais sobre o miocárdio e a vasculatura. A disfunção sistólica sintomática é normalmente encontrada apenas nos casos mais avançados da síndrome de imunodeficiência adquirida. ${ }^{124}$ Trabalhos mais recentes realizados em crianças e adultos jovens comprovaram comprometimento do strain longitudinal de VD e VE, ainda em pacientes assintomáticos e com fração de ejeção do VE normal. Diante desses resultados, Naami et al. sugeriram, em 2016, a incorporação do estudo da deformação miocárdica aos ecocardiogramas dos pacientes pediátricos com HIV, com o objetivo de identificar pacientes com disfunção subclínica e com maior risco cardiovascular. ${ }^{125}$

Em um estudo que incluiu adolescentes e adultos jovens com talassemia submetidos a múltiplas transfusões, Chen et al. ${ }^{126}$ identificaram correlação negativa entre a ferritina sérica e o strain longitudinal do VE. Além disso, mesmo após correção para sexo, idade, nível de ferritina sérica e índice de massa ventricular, o strain longitudinal de VE permaneceu como preditor independente de eventos adversos em pacientes talassêmicos, como insuficiência cardíaca, arritmias e óbito (HR: 6,$05 ; p=0,033) .{ }^{127}$

Investigando crianças e adolescentes portadores de hipertensão pulmonar idiopática (HPI), Okumura et al. comprovaram o valor prognóstico da avaliação seriada do 
strain longitudinal do VD na população pediátrica. Um valor de strain inferior a $-14 \%$ ao ecocardiograma inicial identificou pacientes que evoluíram para transplante pulmonar ou óbito com $100 \%$ de sensibilidade e $54,5 \%$ de especificidade. Concluíram que a deformação miocárdica na HPI pediátrica é ferramenta mais sensível do que os parâmetros convencionais de avaliação da função do VD (TAPSE - do inglês tricuspid annular plane systolic excursion, FAC - do inglês fractional area change, velocidade da onda S tricúspide) para a detecção de pacientes com pior prognóstico. ${ }^{127} \mathrm{Em}$ publicação recente, Hooper et al. ${ }^{128}$ comprovaram a utilidade do strain longitudinal de VD no seguimento clínico da HPI em crianças, demonstrando que os valores de strain apresentam excelente correlação com os valores de BNP - do inglês B-type natriuretic peptide values, no decorrer do tratamento com análogos da prostaciclina. ${ }^{13} \mathrm{~A}$ Tabela 14 apresenta graus de recomendação e níveis de evidência.

\subsection{Strain Ventricular em Cardiopatias Congênitas}

A análise do strain longitudinal do VD em posição subpulmonar provou-se factível e reprodutível na avaliação perioperatória de diferentes cardiopatias congênitas. ${ }^{129}$ Entretanto, na presença de obstrução residual significativa no pós-operatório (PO), parâmetros de avaliação da função sistólica longitudinal do VD, tais como TAPSE, velocidade da onda S e strain de pico sistólico longitudinal, não apresentam adequada correlação com a fração de ejeção obtida pela RM. Em situações com estenose pulmonar residual ou uma combinação de estenose e insuficiência pulmonar, a hipertrofia do VD acarreta predomínio de fibras circunferenciais, alterando o padrão de deformação

Tabela 14 - Recomendações para o strain ventricular em cardiopatias adquiridas na infância ${ }^{20,51,105-128}$

\begin{tabular}{|c|c|c|}
\hline Indicação & $\begin{array}{l}\text { Grau de } \\
\text { recomendação }\end{array}$ & $\begin{array}{l}\text { Nível de } \\
\text { evidência }\end{array}$ \\
\hline Cardiotoxicidade em oncopediatria & Ila & B \\
\hline Miocardites: autoimunes e virais & Ila & $\mathrm{B}$ \\
\hline $\begin{array}{l}\text { Miocardiopatia dilatada: seleção para terapia } \\
\text { de ressincronização }\end{array}$ & lla & B \\
\hline Doença vascular do enxerto pós-TX cardíaco & Ilb & B \\
\hline Rejeição pós-TX cardíaco & $\| l b$ & B \\
\hline Distrofias musculares (p. ex., Duchenne) & Ila & B \\
\hline Doenças de depósito (p. ex., Pompe e MPS) & Ila & B \\
\hline Doença de Kawasaki & Ila & $\mathrm{C}$ \\
\hline Sepse & $\mathrm{Ilb}$ & B \\
\hline Insuficiência renal crônica & $\mathrm{llb}$ & B \\
\hline Infecção pelo HIVIAIDS & Ila & B \\
\hline Anemias crônicas (p. ex., talassemia) & Ila & B \\
\hline Hipertensão pulmonar & Ila & $\mathrm{B}$ \\
\hline
\end{tabular}

dessa câmara, que habitualmente depende mais das fibras longitudinais. ${ }^{130}$ Hayabuchi et al. ${ }^{131}$ avaliaram o strain de pico sistólico circunferencial da parede livre do VD ao corte subcostal, especificamente crianças portadoras de cardiopatias congênitas com sobrecarga pressórica ao VD. Dessa forma, encontraram melhor correlação entre os valores de strain e de fração de ejeção de VD. ${ }^{131}$ Trabalhos envolvendo crianças assintomáticas em pós-operatório tardio de tetralogia de Fallot (T4F) identificaram comprometimento do strain de pico sistólico longitudinal biventricular. Alguns autores encontraram correlação negativa entre o strain de pico sistólico longitudinal de VD e fração de ejeção do VD e fração de regurgitação pulmonar, ambas estimadas pela RM. ${ }^{132}$ Outros trabalhos documentaram correlação negativa entre o strain longitudinal do VE e o grau de regurgitação pulmonar, reforçando a importância da interdependência entre os ventrículos. ${ }^{133}$ Ainda que o estudo da deformação miocárdica consiga detectar disfunção sistólica subclínica nos pacientes operados de T4F que evoluem com regurgitação pulmonar, infelizmente, não há consenso quanto a um valor de corte de strain que permita indicar o melhor momento para a troca valvar pulmonar.

O VD em posição sistêmica também demonstra mudança do padrão de deformação miocárdica, com predomínio de contração das fibras circunferenciais. A redução discreta do strain longitudinal nesta condição representa uma alteração na geometria ventricular direita e não disfunção sistólica real. Trata-se de um mecanismo adaptativo, que faz com que a contratilidade do VD sistêmico se torne semelhante à contratilidade do VE. Por essa razão, trabalhos recentes sugerem uma faixa de valores normais de strain de pico sistólico longitudinal do VD sistêmico que ficam abaixo do esperado para o VD subpulmonar $(-10 \%$ a $-14,5 \%) .{ }^{130}$ Já valores de strain longitudinal de VD inferiores a $-10 \%$ foram associados à ocorrência de eventos adversos, em PO tardio de cirurgia de Senning. ${ }^{134}$

A seleção de pacientes com ventrículo único (VU) para cirurgia de Fontan leva em consideração a resistência vascular pulmonar e a pressão diastólica final ventricular. No entanto, os critérios atuais de indicação mostram-se falhos para uma considerável parcela desses pacientes, que acabam enfrentando complicações e internações prolongadas. Quando associado à resistência vascular pulmonar e à pressão diastólica final ventricular, o strain rate circunferencial préoperatório melhora a estratificação de risco para pacientes com VU candidatos à cirurgia de Fontan, não importando se o ventrículo é de morfologia direita ou esquerda. ${ }^{135}$

No caso da anomalia de Ebstein, o estudo da deformação miocárdica acrescenta pouco à avaliação da função ventricular direita, uma vez que o strain mostra fraca correlação com a fração de ejeção obtida pela RM. ${ }^{136}$

Castaldi et al. ${ }^{137}$ demonstraram utilidade do strain longitudinal do ventrículo esquerdo no diagnóstico de pacientes com obstrução coronariana em PO tardio de correção de origem anômala de coronária esquerda. Um valor de strain $<-14,8 \%$ ao ecocardiograma identificou segmentos miocárdicos com fibrose à RM, com sensibilidade de 92,5\% e especificidade de $93,7 \% .^{137}$ 
Dusenbery et al. ${ }^{138}$ reforçaram essa associação entre redução do strain longitudinal do VE e a presença de fibrose miocárdica, avaliando crianças e adultos jovens com estenose valvar aórtica e com fração de ejeção do VE preservada. ${ }^{138}$ É sabido que adultos com estenose aórtica que apresentam realce tardio à RM com gadolínio e valores reduzidos de strain longitudinal do VE têm maiores taxas de mortalidade após intervenção valvar. ${ }^{138}$ Ver Tabela 15 para graus de recomendação e níveis de evidência.

\subsection{Strain Atrial Direito e Esquerdo em Pediatria}

O estudo da mecânica atrial direita através do speckletracking foi incorporado recentemente à pediatria, surgindo como ferramenta promissora para a detecção de disfunção ventricular direita. Hope et al. ${ }^{139}$ encontraram redução significativa do strain longitudinal de átrio direito em crianças com HPI. O strain atrial mostrou-se mais sensível e específico do que parâmetros convencionais de avaliação da função ventricular direita na identificação dos pacientes com HPI que vieram a apresentar desfechos desfavoráveis (óbito, transplante pulmonar e/ou cardíaco). ${ }^{139}$

Diversos trabalhos têm descrito as implicações clínicas da medida do strain atrial esquerdo pela técnica de speckletracking. O strain de átrio esquerdo, na fase de reservatório, mostrou-se mais acurado na estimativa da pressão diastólica final do VE do que parâmetros ecocardiográficos clássicos como o volume atrial esquerdo e a relação $E / E^{\prime}$, além de correlacionar-se inversamente com os níveis plasmáticos do NT-ProBNP. ${ }^{140}$

\subsection{Perspectivas de Utilização do Strain Ventricular no Feto}

Trabalhos recentes têm sugerido que a análise da deformação miocárdica pode contribuir para a avaliação da
Tabela 15 - Recomendações para o strain ventricular em cardiopatias congênitas ${ }^{129-135,137}$

\begin{tabular}{lcc}
\hline Indicação & $\begin{array}{c}\text { Grau de } \\
\text { recomendação }\end{array}$ & $\begin{array}{c}\text { Nível de } \\
\text { evidência }\end{array}$ \\
\hline $\begin{array}{l}\text { Avaliação da função do VD subpulmonar } \\
\text { (p. ex., T4F) }\end{array}$ & Ilb & $\mathrm{B}$ \\
\hline $\begin{array}{l}\text { Avaliação da função do VD sistêmico (p. ex., } \\
\text { PO de cirurgia de Senning, TCGA) }\end{array}$ & $\| \mathrm{b}$ & $\mathrm{B}$ \\
\hline Avaliação do VU pré-cirurgia de Fontan & $\| \mathrm{b}$ & $\mathrm{B}$ \\
\hline Avaliação do VU pós-cirurgia de Fontan & $\| \mathrm{b}$ & $\mathrm{B}$ \\
\hline Avaliação de VE após correção de OACE & $\| \mathrm{la}$ & $\mathrm{B}$ \\
$\begin{array}{l}\text { Avaliação da função de VE } \\
\text { em estenose aórtica }\end{array}$ & $\| \mathrm{b}$ & $\mathrm{B}$ \\
\hline
\end{tabular}

OACE: origem anômala da coronária esquerda; PO: pós-operatório; T4F: tetralogia de Fallot; TCGA: transposição corrigida das grandes artérias; VD: ventrículo direito; VE: ventrículo esquerdo; VU: ventrículo único.

função sistólica e diastólica biventricular também nos fetos. Como exemplo, Miranda et al. documentaram redução de strain rate diastólico precoce e tardio, no eixo longitudinal de VD e VE, em fetos de mão diabética. Além disso, registraram redução do strain de pico sistólico longitudinal do ventrículo direito, mediante comparação com fetos normais de mesma idade gestacional. Os autores ressaltam que o comprometimento da deformação diastólica ocorreu independentemente da presença de hipertrofia septal. Concluem que o estudo da deformação miocárdica pode detectar alterações subclínicas nos fetos de mãe diabética, antes que os parâmetros ecocardiográficos clássicos sejam capazes de fazê-lo. ${ }^{141}$

\section{Referências}

1. Barberato SH, Romano MMD, Beck ALS, Rodrigues ACT, Almeida ALC, Assunção BMBL, et al. Posicionamento sobre Indicações da Ecocardiografia em Adultos - 2019. Arq Bras Cardiol. 2019; 113(1):135-181.

2. Ferencz C, Rubin JD, McCarter RJ, Brenner JI, Neil CA, Perry LW. Congenital heart disease: prevalence at livebirth. The Baltimore-Washington Infant Study. Am J Epidemiol.1985; 121(1):31-6.

3. Wren C, Richmond S, Donaldson L. Temporal variability in birth prevalence of cardiovascular malformations. Heart. 2000; 83(4):414-9.

4. Yagel S, Weissman A, Rotstein Z, 2 Manor M, Hegesh J, Stoutenbeek P. Congenital heart defects: natural course and in utero development. Circulation. 1997; 96(2):550-5.

5. van Engelen AD, Weijtens O, Brenner JI, Kleinman CS, Copel JA, Stoutenbeek P. et al. Management outcome and follow-up of fetal tachycardia. J Am Coll Cardiol. 1994; 24(5):1371-5.

6. Simpson JM, Sharland GK. Fetal tachycardias: management and outcome of 127 consecutive cases. Heart.1998; 79(6):576-81.

7. Donofrio MT, Moon-Grady AJ, Hornberger LK, Copel JA, Slonsky MS, Abuhamed A, et al. Diagnosis and treatment of fetal cardiac disease: a scientific statement from the American Heart Association. Circulation. 2014; 129(21):2183-242

8. Pedra SRF, Zielinnsky P, Binotto CN, Martins CN, Fonseca ES, Guimarães IC, et al., Sociedade Brasileira de Cardiologia. Diretriz Brasileira de Cardiologia Fetal - 2019. Arq Bras Cardiol. 2019; 112 (5):600-48.

9. Barbosa MM, Nunes MCP, Campos Filho O,Camarozano A, Rabischoffsky A, Maciel BC. , et al., Sociedade Brasileira de Cardiologia. Diretrizes das Indicações da Ecocardiografia. Arq Bras Cardiol. 2009; 936(Supl 3):e265-e302.

10. Noori SS, Seri I. Principles of Developmental Cardiovascular Physiology and Pathophysiology. In: Polin RA.ed. Hemodynamics and Cardiology: Neonatology Questions. 2nd ed. Philadelphia, PA: Elsevier Saunders; 2012.p.3-27.

11. Mertens L, Seri I, Marek J, Arlett0z R, Barker P, McNamara P, et al. Targeted Neonatal Echocardiography in the Neonatal Intensive Care Unit: practice guidelines and recommendations for training. Writing Group of the American Society of Echocardiography (ASE) in collaboration with the European Association of Echocardiography (EAE) and the Association for European Pediatric Cardiologists (AEPC). J Am Soc Echocardiogr. 2011; 24(10):1057-78.

12. Kemper AR, Mahle WT, Martin GR, Cooley WC, Kumar P, Morrow WP. Strategies for implementing screening for critical congenital heart disease. Pediatrics 2011; 128(5):e1259-67. 
13. Afiune JY, Leal SMB, Andrade JL. Avaliação ecocardiográfica das alterações cardiovasculares funcionais do recém-nascido. Rev Bras Ecocardiogr. 2002; 15(2):41-67.

14. Adatia I, Beghetti M. Immediate postoperative care. Cardiol Young. 2009; 19 (Suppl 1):23-7.

15. Badesch DB, Champion HC, Sanchez MA, Hoeper MM, Loyd JE, Manes A. et al. Diagnosis and assessment of pulmonary arterial hypertension. J Am Coll Cardiol. 2009; 54(1 Suppl): S55-66.

16. Cheitlin MD, Armstrong WF, Aurigemma GP. ACC/AHA/ASE 2003 Guideline Update for the Clinical Application of Echocardiography: summary article. A report of the American College of Cardiology/American Heart Association Task Force on Practice Guidelines (ACC/AHA/ASE Committee to Update the 1997 Guidelines for the Clinical Application of Echocardiography). J Am Soc Echocardiogr.2003; 16:1091-110.

17. Lai WW, Geva T, Shirali GS, Frommelt PC, Humes RA, Brook MM, et al Guidelines and standards for performance of a pediatric echocardiogram: a report from the Task Force of the Pediatric Council of the American Society of Echocardiography. J Am Soc Echocardiogr. 2006; 19(12):1413-30.

18. Campbell RM, Douglas PS, Eidem BW, Lai WW, Lopez L, Sachdeva R. ACC/ AAP/AHA/ASE/HRS/SCAI/SCCT/SCMR/SOPE 2014 appropriate use criteria for initial transthoracic echocardiography in outpatient pediatric cardiology: a report of the American College of Cardiology Appropriate Use Criteria Task Force, American Academy of Pediatrics, American Heart Association, American Society of Echocardiography, Heart Rhythm Society, Society for Cardiovascular Angiography and Interventions, Society of Cardiovascular Computed Tomography, Society for Cardiovascular Magnetic Resonance, and Society of Pediatric Echocardiography. J Am Coll Cardiol. 2014; 64(19):2039-60.

19. Leal GN, Silva KF, França CM, Lianza AC, Andrade JL, Kozu K. Subclinical right ventricle systolic dysfunction in chlidhood-onset systemic lupus erythematosus: insights from two-dimensional speckle-tracking echocardiography. Lupus .2015; 24(6):613-20.

20. Leal GN, Silva KF, Lianza AC, Giacomin MF, Andrade JL, Campos LM. Subclinical left ventricular dysfunction in childhood-onset systemic lupus erythematosus: a two-dimensional speckle-tracking echocardiography study. Scand J Rheumatol. 2016 ; 45(3):202-9.

21. Lianza AC, Aikawa NE, Morae JC, Leal GN, Morhy SS, Andrade JL. Long-term evaluation of cardiac function in juvenile idiopathic arthritis under anti-TNF therapy. Clin Exp Rheumatol.2014; 32(5):754-9.

22. Gewitz MH, Baltimore RS, Tani LY, Sable CA, Shulman ST, Carapetis J. Revision of the Jones Criteria for the diagnosis of acute rheumatic fever in the era of Doppler echocardiography: a scientific statement from the American Heart Association. Circulation. 2015; 131(20):1806-18.

23. Ryan TD, Nagarajan R, Godown J. Pediatric cardio-oncology: development of cancer treatment-related cardiotoxicity and the therapeutic approach to affected patients. Curr Treat. Options Oncol. 2019; 20(7):56.

24. Flynn JT, Kaelber DC, Baker-Smith CM, Blowey D, Carroll AE, Daniels SR. Clinical practice guideline for screening and management of high blood pressure in children and adolescents. Pediatrics. 2017; 140(3):1-72.

25. Anuardo P, Verdier M, Gormezano NW, Ferreira GR, Leal GN, Lianza A. Subclinical pulmonary hypertension in childhood systemic lupus erythematosus associated with minor disease manifestations. Pediatr Cardiol. 2007; 38(2):234-9.

26. Patel MD, Breatnach CR, James AT, Choudhry S, McNamara PJ, Jain A, et al. Echocardiographic assessment of right ventricular afterload in preterm infants: maturational patterns of pulmonary artery acceleratio time over the first year of age and implications for pulmonary hypertension. J Am Soc Echocardiogr. 2019; 32(7):884-894

27. Koestenberger M, Apitz C, Abdul-Khaliq H, Hansmann G. Transthoracic echocardiography for the evaluation of children and adolescents with suspected or confirmed pulmonary hypertension. Expert consensus statement on the diagnosis and treatment of paediatric pulmonary hypertension. The European Paediatric Pulmonary Vascular Disease Network, endorsed by ISHLT and D6PK. Heart 2016; 102 ( Suppl 2):ii14-22.
28. Lipshultz SE, Wilkinson JD, Thompson B, Cheng I,Briston DA, Shearer WT. Cardiac effects of highly active antiretorviral therapy in perinatally HIV-infected children: The CHAART-@ Study. J Am Coll Cardiol. 2017; 70(18):2240-7.

29. Kindel SJ, Hsu HH, Hussain T, Johnson JN, McMahon Cj, Kutty S, et al. Multimodality noninvasive imaging in the monitoring of pediatric heart transplantation. J Am Soc Echocardiogr. 2017; 30(9):859-70.

30. Platts DG, Sedgwick JF, Burstow DJ, Mullany DV, Fraser JF. The role of echocardiography in the management of patients supported by extracorporeal membrane oxygenation. J Am Soc Echocardiogr. 2012; 25:131-41.

31. Newburger JW, Takahashi M, Gerver MA. Diagnosis, treatment, and longterm management of Kawasaki disease: a statement for health professionals from the Committee on Rheumatic Fever, Endocarditis and Kawasaki Disease, Council on Cardiovascular Disease in the Young, American Heart Association. Circulation.2004; 110(17):2747-71.

32. Gilboa SM, Devine OJ, Kucik JE, et al. Congenital Heart Defects in the United States: Estimating the Magnitude of the Affected Population in 2010. Circulation 2016; 134(2): 101-9

33. Di Nardo JA. Grown-up congenital heart (GUCH) disease: an evolving global challenge. Ann Card Anaesth. 2008; 11(1):3-5.

34. Van der Bom T, Bouma BJ, Meijboom FJ,Zwinderman AH, Mulder BJ, et al. The prevalence of adult congenital heart disease, results froma asystematic review and evidence based calculation. Am Heart J. 2012; 164(4):568-75

35. Thakkar AN, Chinnadurai P, Lin CH. Adult congenital heart disease: magnitude of the problem. Curr Opin Cardiology 2017; 32(5):467-74.

36. Baumgartner $\mathrm{H}$, Bonhoeffer $\mathrm{P}$, De Groot NM et al. ESC Guidelines for the management of grown-up congenital heart disease (new version 2010). Eur Heart J.2010; 31(23):2915-57.

37. Simpson J, Lopez L, Acar P, et al. Three-dimensional Echocardiography in Congenital Heart Disease: An Expert Consensus Document from the European Association of Cardiovascular Imaging and the American Society of Echocardiography. J Am Soc Echocardiogr. 2017; 30:1-27.

38. Graziani F, Delogu AB. Evaluation of Adults With Congenital Heart Disease. World J Pediatr Congenit Heart Surg. 2016; 7(2):185-91.

39. Bhatt AB, Foster E, Kuehl K, Alpert J, Brabeck S, Crumb S, et al. Congenital heart disease in the older adult: a scientific statement from the American Heart Association. Circulation. 2015; 131(21):1884-931.

40. Group TTACW, Douglas PS, Khandheria B et al. ACCF/ASE/ACEP/ASNC/ SCAI/SCCT/SCMR 2007 appropriateness criteria for transthoracic and transesophageal echocardiography: a report of the American College of Cardiology Foundation Quality Strategic Directions Committee Appropriateness Criteria Working Group, American Society of Echocardiography, American College of Emergency Physicians, American Society of Nuclear Cardiology, Society for Cardiovascular Angiography and Interventions, Society of Cardiovascular Computed Tomography, and the Society for Cardiovascular Magnetic Resonance. Endorsed by the American College of Chest Physicians and the Society of Critical Care Medicine. J Am Soc Echocardiogr. 2007; 20:787-805

41. Stout KK, Daniels CJ, Aboulhosn JA, Bozkurt B, Broberg CS, Colman JM, et al. $2018 \mathrm{AHA} / \mathrm{ACC}$ Guideline for the management of adults with congenital heart disease. A report of the American College of Cardiology/American Heart Association Task Force on Clinical Practice Guidelines. Circulation 2019; 139(14):e698-e800.

42. Silversides CK, Kiess M, Beauchesne L, Bradley T, Broberg CS, Colman JM. et al. Canadian Cardiovascular Society 2009 Consensus Conference on the management of adults with congenital heart disease: outflow tract obstruction, coarctation of the aorta, tetralogy of Fallot, Ebstein anomaly and Marfan's syndrome. Canad J Cardiol. 2010; 26(3):e80-97.

43. Silversides CK, Salehian O, Oechslin E, Schwerzmann M, Muhll IV, Klairy P, et al. Canadian Cardiovascular Society 2009 Consensus Conference on the management of adults with congenital heart disease: complex congenital cardiac lesions. Can J Cardiol.2010; 26(3):e98-117. 
44. Hiratzka LF, Bakris GL, Beckman JA, et al. 2010 ACCF/AHA/AATS/ACR/ASA/ SCA/SCAI/SIR/STS/SVM Guidelines for the diagnosis and management of patients with thoracic aortic disease. A Report of the American College of Cardiology Foundation/American Heart Association Task Force on Practice Guidelines, American Association for Thoracic Surgery, American College of Radiology,American Stroke Association, Society of Cardiovascular Anesthesiologists, Society for Cardiovascular Angiography and Interventions, Society of Interventional Radiology, Society of Thoracic Surgeons, and Society for Vascular Medicine. J Am Coll Cardiol.2010; 55:e27-e129.

45. Puchalski MD, Lui GK, Miller-Hance WC,Brook MM, Young LT, Bhat A. Guidelines for Performing a Comprehensive Transesophageal Echocardiographic Examination in Children and All Patients with Congenital Heart Disease: Recommendations from the American Society of Echocardiography. J Am Soc Echocardiogr. 2019; 32(2):173-215.

46. Bettex DA, Pretre R, Jenni R, Schmid ER. Cost-effectiveness of routine intraoperative transesophageal echocardiography in pediatric cardiac surgery: a 10-year experience. Anesthes Analg. 2005; 100(5):1271-5.

47. Rigby ML. Transoesophageal echocardiography during interventional cardiac catheterisation in congenital heart disease. Heart. 2001; 86(Suppl 2):II23-9.

48. Pellikka PA, Nagueh SF, Elhendy AA, Kuehl CA, Sawada SG, American Society of E. American Society of Echocardiography recommendations for performance, interpretation, and application of stress echocardiography. J Am Soc Echocariogr. 2007; 20(9):1021-41.

49. Sicari R, Nihoyannopoulos P, Evangelista A, Kasprzak J, Lancellotti P, Poldermans D. Stress Echocardiography Expert Consensus Statement-Executive Summary: European Association of Echocardiography (EAE) (a registered branch of the ESC). Eur Heart J. 2009; 30(3):278-89.

50. Noto N, Kamiyama H, Karasawa K,Ayusawa M, Sumitomo N, Okada T, et al. Long-term prognostic impact of dobutamine stress echocardiography in patients with Kawasaki disease and coronary artery lesions: a 15-year follow-up study. J Am Coll Cardiol.2014; 63(4):337-44.

51. McCrindle BW, Rowley AH, Newburger JW et al. Diagnosis, Treatment, and Long-Term Management of Kawasaki Disease: A Scientific Statement for Health Professionals From the American Heart Association. Circulation. 2017; 135(17):e927-e999.

52. Dipchand Al, Bharat W, Manlhiot C, Safi M, Lobach NE, McCrindle BW. A prospective study of dobutamine stress echocardiography for the assessment of cardiac allograft vasculopathy in pediatric heart transplant recipients. Pediat Transplant. 2008; 129(5):570-6.

53. Ou P, Khraiche D, Celermajer DS,Agnoletti G, Sang KH, Thalabard JC, et al. Mechanisms of coronary complications after the arterial switch for transposition of the great arteries. The Journal of thoracic and cardiovascular surgery 2013; 145(5):1263-9.

54. Hui L, Chau AK, Leung MP, Chiu CS, Cheung YF. Assessment of left ventricular function long term after arterial switch operation for transposition of the great arteries by dobutamine stress echocardiography. Heart. 2005; 91(1):68-72.

55. Brothers JA, FrommeltMA, Jaquiss RDB, Myerburg RJ, Fraser CD Jr., Tweddell JS. Expert consensus guidelines: Anomalous aortic origin of a coronary artery. J Thorac Cardiovasc Surg. 2017; 153(6):1440-57.

56. Lancellotti P, Pellikka PA, Budts W, et al. The Clinical Use of Stress Echocardiography in Non-Ischaemic Heart Disease: Recommendations from the European Association of Cardiovascular Imaging and the American Society of Echocardiography. J Am Soc Echocardiogr. 2017; 30:101-38.

57. Cifra B, Dragulescu A, Brun H,McCrindle BW, Dipchand A, MertensL, et al. Left ventricular myocardial response to exercise in children after heart transplant. The J Heart Lung Transplant. 2014; 33(2):1241-7.

58. De Meester P, Buys R, Van De Bruaene A, Gabroels C, Voigt JU, Vanhees $\mathrm{L}$, et al. Functional and haemodynamic assessment of mild-to-moderate pulmonary valve stenosis at rest and during exercise. Heart. 2014; 100(17):1354-9.

59. Hasan BS, Lunze Fl, McElhinney DB, Stantchevo E, Bron DW, Rhodes J. et al. Exercise stress echocardiographic assessment of outflow tract and ventricular function in patients with an obstructed right ventricular-to-pulmonary artery conduit after repair of conotruncal heart defects. Am J Cardiol. 2012; 110(10):1527-33.

60. Chen CK, Cifra B, Morgan G), Sarcola T, Slorach C, Wei H. Left Ventricular Myocardial and Hemodynamic Response to Exercise in Young Patients after Endovascular Stenting for Aortic Coarctation. J Am Soc Echocardiogr. 2016; 29(3):237-46.

61. Ait-Ali L, Siciliano V, Passino C, Molinoro S, Pasanisi E, Sicari R, et al. Role of stress echocardiography in operated fallot: feasibility and detection of right ventricular response. J Am Soc Echocardiogr.2014; 27(12): 1319-28.

62. Oosterhof T, Tulevski, II, Roest AA,Steendijk P, Vliegen HW, Wall E, et al. Disparity between dobutamine stress and physical exercise magnetic resonance imaging in patients with an intra-atrial correction for transposition of the great arteries. J Cardiovasc Magn Reson. 2005; 7(2):383-9.

63. Vogt M, Kuhn A, Wiese J, Eicken A, Hess J, Vogel M. Reduced contractile reserve of the systemic right ventricle under Dobutamine stress is associated with increased brain natriuretic peptide levels in patients with complete transposition after atrial repair. Eur J Echocardiogr.2009; 10(5):691-4.

64. Klewer SE, Goldberg SJ, Donnerstein RL, Berg RA, Hutter JJ Jr. Dobutamine stress echocardiography: a sensitive indicator of diminished myocardial function in asymptomatic doxorubicin-treated long-term survivors of childhood cancer. J Am Coll Cardiol. 1992; 19(2):394-401.

65. Ryerson AB, Border WL, Wasilewski-Masker K, Goodman M, Meachman L, Austin $\mathrm{H}$. Assessing anthracycline-treated childhood cancer survivors with advanced stress echocardiography. Pediatr Blood Cancer. 2015; 62(3):502-8.

66. Cifra B, Dragulescu A, Border WL, Mertens L. Stress echocardiography in paediatric cardiology. Eur Heart J Cardiovasc Imag.2015; 16(10):1051-9.

67. Hecht HS, DeBord L, Sotomayor N, Shaw R, Dunlap R, Ryan C. Supine bicycle stress echocardiography: peak exercise imaging is superior to postexercise imaging. J Am Soc Echocardiogr.1993; 6(3 pt 1):265-71.

68. Simpson JM. Real-time three-dimensional echocardiography of congenital heart disease using a high frequency paediatric matrix transducer. Eur J Echocardiogr. 2008; 9(2):222-4.

69. van der Zwaan HB, Helbing WA, McGhie JS, Geleijnse ML, Luijnenburg SE, Hesselink JW. Clinical value of real-time three-dimensional echocardiography for right ventricular quantification in congenital heart disease: validation with cardiac magnetic resonance imaging. J Am Soc Echocardiogr; 2010; 23(2):134-40.

70. Friedberg MK, Su X, Tworetzky W, Soriano BD, Powell AJ, Marx GR. Validation of 3D echocardiographic assessment of left ventricular volumes, mass, and ejection fraction in neonates and infants with congenital heart disease: a comparison study with cardiac MRI. Circ Cardiovasc Imaging 2010; 3(6):735-42.

71. Takahashi K, Mackie AS, Thompson R,Naami G, Inage A, Rebyka IM. Quantitative real-time three-dimensional echocardiography provides new insight into the mechanisms of mitral valve regurgitation post-repair of atrioventricular septal defect. J Am Soc Echocardiogr 2012; 25(11):1231-44.

72. Simpson J, Lopez L, Acar P, Friedberg MK, Khoo NS, Ko HH, et al. Threedimensional Echocardiography in Congenital Heart Disease: An Expert ConsensusDocument from the European Association of Cardiovascular Imaging and the American Society of Echocardiography J Am Soc Echocardiogr. 2017; 30(1):1-27.

73. McGhie JS, van den Bosch AE, HaarmanMG, Ren B, Roos-Hesselink JW, Witsenburg M, et al. Characterization of atrial septal defect by simultaneous multiplane two-dimensional echocardiography. Eur Heart J Cardiovasc Imaging 2014; 15(10):1145-51.

74. Pushparajah K, MillerOI, Simpson JM. 3Dechocardiography of the atrial septum: anatomical features and landmarks for the echocardiographer. JACC Cardiovasc Imaging 2010; 3(9):981-4.

75. Bharucha T, Anderson RH, Lim ZS, Vettukattil JJ. Multiplanar review of threedimensional echocardiography gives new insights into the morphology of Ebstein's malformation. Cardiol Young. 2010; 20(1):49-53. 
76. van Noord PT, Scohy TV, McGhie J, Bogers AJJC. Three-dimensional transesophageal echocardiography in Ebstein's anomaly. Interact Cardiovasc Thorac Surg. 2010; 10(5):836-7.

77. Roberson DA, Cui W, Patel D, Tsang W, Sugeng L, Weinert L, et al. Threedimensional transesophageal echocardiography of atrial septal defect: a qualitative and quantitative anatomic study. J Am Soc Echocardiogr. 2011; 24(6):600-10.

78. Sivakumar K, Singhi A, Pavithran S. Enface reconstruction of VSD on RV septal surface using real-time 3D echocardiography. JACC Cardiovasc Imaging. 2012; 5(11):1176-80

79. Kutty S, Smallhorn JF. Evaluation of atrioventricular septal defects by threedimensional echocardiography: benefits of navigating the third dimension. J Am Soc Echocardiogr. 2012; 25(9):932-44

80. Marechaux S, Juthier F, Banfi C, Vincentelli A, Prat A, Ennezat P-V. Illustration of the echocardiographic diagnosis of subaortic membrane tenosis in adults: surgical and live three-dimensional transoesophageal findings. Eur J Echocardiogr. 2011; 12(1):E2.

81. Noel CV, Choy RM, Lester IR, Soriano BD. Accuracy of matrix-array threedimensional echocardiographic measurements of aortic root dilation and comparison with two-dimensional echocardiography in pediatric patients. J Am Soc Echocardiogr. 2012; 25(3):287-93.

82. Hlavacek A, Lucas J, Baker H, Chessa K, Shirali G. Feasibility and utility of three-dimensional color flow echocardiography of the aortic arch: The "echocardiographic angiogram". Echocardiography. 2006; 23:860-4.

83. Valente AM, Cook S, Festa P, Ko HH, Krishnamurthy R, Taylor AM, et al Multimodality imaging guidelines for patients with repaired tetralogy of fallot: a report from the American Society of Echocardiography: developed in collaboration with the Society for Cardiovascular Magnetic Resonance and the Society for Pediatric Radiology. J Am Soc Echocardiogr. 2014; 27(5):111-41.

84. Abadir S, Leobon B, Acar P. Assessment of tricuspid regurgitation mechanism by three-dimensional echocardiography in an adult patient with congenitally corrected transposition of the great arteries. Arch Cardiovasc Dis. 2009; 102(5):459-60.

85. Del Pasqua A, Sanders SP, De Zorzi A, Toscano A, lacobelli R, Pierli C, et al. Impact of three-dimensional echocardiography in complex congenital heart defect cases: the surgical view. Pediatr Cardiol. 2009; 30(3):293-300.

86. Pushparajah K, Barlow A, Tran V-H, Miller OI, Zidere V, Vaidyanathan B, et al. A systematic three-dimensional echocardiographic approach to assist surgical planning in double outlet right ventricle. Echocardiography. 2013; 30(2):234-8.

87. Saric M, Perk G, Purgess JR, Kronzon I. Imaging atrial septal defects by realtime three-dimensional transesophageal echocardiography: stepby-step approach. J Am Soc Echocardiogr.(11):1128-35.

88. Charakida M, Qureshi S, Simpson JM. 3D echocardiography for planning and guidance of interventional closure of VSD. JACC Cardiovasc Imaging. 2013; 6(1):120-3.

89. Giannakoulas G, Thanopoulos V. Three-dimensional transesophageal echocardiography for guiding percutaneous fontan fenestration closure. Echocardiography. 2014; 31(7):e230-1.

90. Raslan S, Nanda NC, Lloyd L, Khairnar P, Reilly SD, Holman WL. Incremental value of live/real time three-dimensional transesophageal echocardiography over the two-dimensional technique in the assessment of sinus of valsalva aneurysm rupture. Echocardiography. 2011; 28(8):918-20.

91. Cavalcante JL, Rodriguez LL, Kapadia S, Tuzcu EM, Stewart WJ. Role of echocardiography in percutaneous mitral valve interventions. JACC Cardiovasc Imaging. 2012; 5(7):733-46.

92. Cua CL, Kollins K, Roble S, Holzer RJ. Three-dimensional image of a baffle leak in a patient with a mustard operation. Echocardiography. 2014; 31(10):e315-6.

93. Deoring M, Braunschweig F, Eitel C, Gaspar T, Wetzel U, Nitsche B, et al. Individually tailored left ventricular lead placement: lessons from multimodality integration between three-dimensional echocardiography and coronary sinus angiogram. Europace. 2013; 15(5):718-27.

94. Mishra J, Puri HP, Hsiung MC, Misra S, Khairnar P, Laxmi Gollamudi B, et al Incremental value of live/real time three-dimensional over two dimensional transesophageal echocardiography in the evaluation of right coronary artery fistula. Echocardiography. 2011; 28(7):805-8.

95. Renella P, Marx GR, Zhou J, Gauvreau K, Geva T. Feasibility and reproducibility of three-dimensional echocardiographic assessment of right ventricular size and function in pediatric patients. J Am Soc Echocardiogr. $2014 ; 27(8): 903-10$.

96. Maffessanti F, Muraru D, Esposito R, Gripari P, Ermacora D, Santoro C, et al. Age-, body size-, and sex-specific reference values for right ventricular volumes and ejection fraction by three-dimensional echocardiography: a multicenter echocardiographic study in 507 healthy volunteers. Circ Cardiovasc Imaging. 2013; 6(5):700-10.

97. Leibundgut G, Rohner A, Grize L, Bernheim A, Kessel-Schaefer A, Bremerich J, et al. Dynamic assessment of right ventricular volumes and function by real-time three-dimensional echocardiography: a comparison study with magnetic resonance imaging in 100 adult patients. J Am Soc Echocardiogr. 2010; 23(2):116-26.

98. Dragulescu A, Mertens LL. Developments in echocardiographic techniques for the evaluation of ventricular function in children. Arch Cardiovasc Dis. 2010; 103(11-12):603-14.

99. Mor-Avi V, Lang RM, Badano LP, Belohlavek M, Cardim NM, Derumeaux $\mathrm{G}$, et al. Current and evolving echocardiographic techniques for the quantitative evaluation of cardiac mechanics: ASE/EAE consensus statement on methodology and indications endorsed by the Japanese Society of Echocardiography. Eur J Echocardiogr. 2011; 12(3):167-205.

100. Collier P, Phelan D, Klein A. Test in Context: Myocardial Strain Measured by Speckle-Tracking Echocardiography. J Am Coll Cardiol. 2017; 69(8):1043-56.

101. Forsey J, Friedberg MK, Mertens L. Speckle tracking echocardiography in pediatric and congenital heart disease. Echocardiography. 2013; 30(4):447-59.

102. Levy PT, Sanchez Mejia AA, Machefsky A, Fowler S, Holland MR, Singh GK. Normal ranges of right ventricular systolic and diastolic strain measures in children: a systematic review and meta-analysis. J Am Soc Echocardiogr. 2014; 27(5):549-60.

103. Levy PT, Machefsky A, Sanchez AA, Patel MD, Rogal S, Fowler S, et al. Reference Ranges of Left Ventricular Strain Measures by Two-Dimensional Speckle-TrackingEchocardiography in Children: A Systematic Review and Meta-Analysis. J Am Soc Echocardiogr. 2016; 29(3):209-25.

104. Kutty S, Padiyath A, Li L, Peng Q, Rangamani S, Schuster A, et al.

105. Larsen CM, Mulvagh SL. Cardio-oncology: what you need to know now for clinical practice and echocardiography. Echo Res Pract. 2017; 4(1): R33-R41.

106. Plana JC, Galderisi M, Barac A, Ewer MS, Ky B, Scherrer-Crosbie M, et al. Expert consensus for multimodality imaging evaluation of adult patients during and after cancer therapy: a report from the American Society of Echocardiography and the European Association of Cardiovascular Imaging. Eur Heart J Cardiovasc Imaging. 2014; 15(10):1063-93.

107. Negishi T, Thavendiranathan P, Negishi K, Marwick TH; SUCCOUR investigators. Rationale and Design of the Strain Surveillance of Chemotherapy for Improving Cardiovascular Outcomes (SUCCOUR) Trial. JACC Cardiovasc Imaging. 2018. pii: S1936-878X (18)30302-4.

108. Agha H, Shalaby L, Attia W, Abdelmohsen G, Aziz OA, Rahman MY. Early Ventricular Dysfunction After Anthracycline Chemotherapy in Children. Pediatr Cardiol. 2016; 37(3):537-44.

109. Leal GN, Diniz MF, Brunelli J, Lianza AC, Sallum AM, Silva CA. What are the benefits of two-dimensional speckle tracking echocardiography for diagnosis and treatment follow-up of childhood-onset systemic lupus erythematosus myocarditis? Rev Assoc Med Bras. 2016; 62(6):490-3. 
110. Caspar T, Germain P, El Ghannudi S, Morel O, Samet H, Trinh A, et al. Acute Myocarditis Diagnosed by Layer-Specific 2D Longitudinal Speckle Tracking Analysis. Echocardiography. 2016; 33(1):157-8.

111. Forsha D, Slorach C, Chen CK, Sherman A, Mertens L, Barker P, et al. Patterns of Mechanical Inefficiency in Pediatric Dilated Cardiomyopathy and Their Relation to LeftVentricular Function and Clinical Outcomes. J Am Soc Echocardiogr. 2016; 29(3):226-36

112. Boruta RJ, Miyamoto SD, Younoszai AK, Patel SS, Landeck BF 2nd. Worsening in Longitudinal Strain and Strain Rate Anticipates Development of pediatric transplant coronary artery vasculopathy as soon as one year following transplant. Pediatr Cardiol. 2018; 39(1):129-39.

113. Badano LP, Miglioranza MH, Edvardsen T, Colafranceschi AS, Muraru D, Bacal F, Nieman K, et al. European Association of Cardiovascular Imaging Cardiovascular Imaging Department of the Brazilian Society of Cardiology recommendations for the use of cardiac imaging to assess and follow patients after heart transplantation. Eur Heart J Cardiovasc Imaging. 2015; 16(9):919-48.

114. Gursu HA, Varan B, Sade E, Erdogan I, Sezgin A, Aslamaci S. Evaluation of Acute Rejection by Measuring Strain and Strain Rate in Children with Heart Transplant: A Preliminary Report. Exp Clin Transplant. 2017; 15(5):561-6.

115. Dandel M, Hetzer R. Post-transplant surveillance for acute rejection and allograft vasculopathy by echocardiography: Usefulness of myocardial velocity and deformation imaging. J Heart Lung Transplant. 2017; 36(2):117-31.

116. Jo WH, Eun LY, Jung JW, Choi JY, Gang SW. Early marker of myocardial deformation in children with Duchenne Muscular Dystrophy assessed using echocardiographic myocardial strain analysis. Yonsei Med J. 2016 ; 57(4):900-4.

117. Duboc D, Meune C, Pierre B, Wahbi K, Eymard B, Toutain A, etal. Perindopril preventive treatment on mortality in Duchenne muscular dystrophy: 10 years' follow-up. Am Heart J. 2007; 154(3):596-602.

118. Borgia F, Pezzullo E, Schiano Lomoriello V, Sorrentino R, Lo ludice $\mathrm{F}$, Cocozza S, et al. Myocardial deformation in pediatric patients with mucopolysaccharidoses: A two-dimensional speckle tracking echocardiography study. Echocardiography. 2017; 34(2):240-9.

119. Chen CA, Chien YH, Hwu WL, et al. Left ventricular geometry, global function, and dyssynchrony in infants and children with Pompe cardiomyopathy undergoing enzyme replacement therapy. J Cardiac Fail. 2011; 17(11):930-6.

120. Avula S, Nguyen TM, Marble M, Lilje C. Cardiac response to enzyme replacement therapy in infantile Pompe disease with severe hypertrophic cardiomyopathy. Echocardiography. 2017; 34(4):621-4.

121. McCandless RT, Minich LL, Wilkinson SE, McFadden ML, Tani LY, Menon SC. Myocardial strain and strain rate in Kawasaki disease. Eur Heart J Cardiovasc Imaging. $2013 \mathrm{Nov}$; 14(11):1061-8.

122. Haileselassie B, Su E, Pozios I, Fiskum T, Thompson R, Abraham T. Strain echocardiography parameters correlate with disease severity in children and infants with sepsis. Pediatr Crit Care Med. 2016 May; 17(5):383-90.

123.van Huis M, Schoenmaker NJ, Groothoff JW, van der Lee JH, van Dyk M Gewillig M, et al. Impaired longitudinal deformation measured by speckletracking echocardiography in children with end-stage renal disease. Pediatr Nephrol. 2016; 31(9):1499-508.

124. Starc TJ, Lipshultz SE, Easley KA, Kaplan S, Bricker JT, Colan SD, etal. Incidence of cardiac abnormalities in children with human immunodeficiency virus infection: The prospective P2C2 HIV study. J Pediatr. 2002 ; 141(3):327-34.

125. Al-Naami G, Kiblawi F, Kest H, Hamdan A, Myridakis D. Cardiac mechanics in patients with human immunodeficiency virus: a study of systolic myocardial deformation in children and young adults. Pediatr Cardiol. 2014; 35(6): 1046-51.
126. Chen MR, Ko HS, Chao TF, Liu HC, Kuo JY, Bulwer BE, et al. Relation of myocardial systolic mechanics to serum ferritin level as a prognosticator in thalassemia patients undergoing repeated transfusion. Echocardiography. 2015; 32(1):79-88.

127. Okumura K, Humpl T, Dragulescu A, Mertens L, Friedberg MK. Longitudinal assessment of right ventricular myocardial strain in relation to transplantfree survival in children with idiopathic pulmonary hypertension. J Am Soc Echocardiogr. 2014; 27(12):1344-51.

128. Hopper RK, Wang Y, DeMatteo V, Santo A, Kawut SM, Elci OU, et al. Right ventricular function mirrors clinical improvement with use of prostacyclin analogues in pediatric pulmonary hypertension. Pulm Circ. 2018; 8(2):2045894018759247.

129. Forsey J, Friedberg MK, Mertens L. Speckle tracking echocardiography in pediatric and congenital heart disease. Echocardiography. 2013; 30(4):447-59.

130. Karsenty C, Hadeed K, Dulac Y, Semet F, Alacoque X, Breinig S, et al. Twodimensional right ventricular strain by speckle tracking for assessment of longitudinal right ventricular function after pediatric congenital heart disease surgery. Arch Cardiovasc Dis. 2017; 110(3):157-66.

131. Hayabuchi Y, Sakata M, Kagami S. Right ventricular myocardial deformation patterns in children with congenital heart diseaseassociated with right ventricular pressure overload. Eur Heart J Cardiovasc Imaging. 2015; 16(8):890-9.

132. Yim D, Mertens L, Morgan CT, Friedberg MK, Grosse-Wortmann L, Dragulescu A. Impact of surgical pulmonary valve replacement on ventricular mechanics in children with repaired tetralogy of Fallot. Int J Cardiovasc Imaging. 2017; 33(5):711-20.

133. Sabate Rotes A, Bonnichsen CR, Reece CL, Connolly HM, Burkhart HM, Dearani JA, et al. Long-term follow-up in repaired tetralogy of Fallot: can deformation imaging help identify optimal timing of pulmonary valve replacement? J Am Soc Echocardiogr. 2014; 27(12):1305-10.

134. Iriart X, Roubertie F, Jalal Z, Thambo JB. Quantification of systemic right ventricle by echocardiography. Arch Cardiovasc Dis. 2016; 109(2):120-7.

135.Park PW, Atz AM, Taylor CL, Chowdhury SM. Speckle-Tracking Echocardiography Improves Pre-Operative Risk Stratification Before the Total Cavopulmonary Connection. J Am Soc Echocardiogr. 2017; 30(5):478-84.

136. Kühn A, Meierhofer C, Rutz T, Rondak IC, Röhlig C, Schreiber C, et al. Non - volumetric echocardiographic indices and qualitative assessment of right ventricular systolic function in Ebstein's anomaly: comparison with CMRderived ejection fraction in 49 patients. Eur Heart J Cardiovasc Imaging. 2016; 17(8):930-5.

137. Castaldi B, Vida V, Reffo E, Padalino M, Daniels Q, Stellin G, et al. Speckle Tracking in ALCAPA Patients After Surgical Repair as Predictorof Residual Coronary Disease. Pediatr Cardiol. 2017; 38(4):794-800.

138. Dusenbery SM, Lunze FI, Jerosch-Herold M, Geva T, Newburger JW, Colan $\mathrm{SD}$, et al. Left Ventricular Strain and Myocardial Fibrosis in Congenital Aortic Stenosis. Am J Cardiol. 2015; 116(8):1257-62.

139. Hope KD, Calderón Anyosa RJC, Wang Y, Montero AE, Sato T, Hanna BD, et al. Right atrial mechanics provide useful insight in pediatric pulmonary hypertension. Pulm Circ. 2018; 8(1):2045893218754852

140. Cameli M, Mandoli GE, Loiacono F, Dini FL, Henein M, Mondillo S. Left atrial strain: a new parameter for assessment of left ventricular filling pressure. Heart Fail Rev. 2016; 21(1):65-76.

141. Miranda JO, Cerqueira RJ, Ramalho C, Areias JC, Henriques-Coelho T. Fetal Cardiac Function in Maternal Diabetes: A Conventional and Speckle-Tracking Echocardiographic Study. J Am Soc Echocardiogr. 2018; 31(3):333-41 